\title{
Classical irregular blocks, Hill's equation and PT-symmetric periodic complex potentials
}

\author{
Marcin Piatek ${ }^{a, c}$ and Artur R. Pietrykowski ${ }^{b, c}$ \\ ${ }^{a}$ Institute of Physics, University of Szczecin, \\ ul. Wielkopolska 15, Szczecin, 70-451 Poland \\ ${ }^{b}$ Institute of Theoretical Physics, University of Wroctaw, \\ pl. M. Borna, Wroctaw, 950-204 Poland \\ ${ }^{c}$ Bogoliubov Laboratory of Theoretical Physics, Joint Institute for Nuclear Research, \\ Dubna, Moscow region, 141980 Russia \\ E-mail: piatek@fermi.fiz.univ.szczecin.pl, pietrie@theor.jinr.ru
}

ABSTRACT: The Schrödinger eigenvalue problems for the Whittaker-Hill potential $Q_{2}(x)=$ $\frac{1}{2} h^{2} \cos 4 x+4 h \mu \cos 2 x$ and the periodic complex potential $Q_{1}(x)=\frac{1}{4} h^{2} \mathrm{e}^{-4 i x}+2 h^{2} \cos 2 x$ are studied using their realizations in two-dimensional conformal field theory (2dCFT). It is shown that for the weak coupling (small) $h \in \mathbb{R}$ and non-integer Floquet parameter $\nu \notin \mathbb{Z}$ spectra of hamiltonians $\mathcal{H}_{i}=-\mathrm{d}^{2} / \mathrm{d} x^{2}+Q_{i}(x), i=1,2$ and corresponding two linearly independent eigenfunctions are given by the classical limit of the "single flavor" and "two flavors" $\left(N_{f}=1,2\right)$ irregular conformal blocks. It is known that complex nonhermitian hamiltonians which are $\mathrm{PT}$-symmetric (= invariant under simultaneous parity $\mathrm{P}$ and time reversal $\mathrm{T}$ transformations) can have real eigenvalues. The hamiltonian $\mathcal{H}_{1}$ is PT-symmetric for $h, x \in \mathbb{R}$. It is found that $\mathcal{H}_{1}$ has a real spectrum in the weak coupling region for $\nu \in \mathbb{R} \backslash \mathbb{Z}$. This fact in an elementary way follows from a definition of the $N_{f}=1$ classical irregular block. Thus, $\mathcal{H}_{1}$ can serve as yet another new model for testing postulates of PT-symmetric quantum mechanics.

KeYwords: Conformal and W Symmetry, Discrete Symmetries, Field Theories in Lower Dimensions, Supersymmetric gauge theory

ARXIV EPRINT: 1604.03574 


\section{Contents}

1 Introduction 1

2 Quantum and classical $N_{f}=1,2$ irregular blocks $\quad 4$

2.1 Regular and $N_{f}=1,2$ irregular blocks 4

$\begin{array}{ll}2.2 \text { Null vector decoupling equations } & 10\end{array}$

3 Classical limit of $N_{f}=1,2$ NVD equations 12

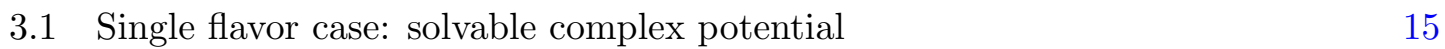

$\begin{array}{ll}3.2 \text { Two flavors case: Whittaker-Hill equation } & 17\end{array}$

4 Conclusions 18

$\begin{array}{ll}\text { A Floquet's theorem and band structure of spectrum } & 19\end{array}$

\section{Introduction}

The Hill's equation is a second-order linear ordinary differential equation of the (Schrödinger-like) form:

$$
-\psi^{\prime \prime}(x)+Q(x) \psi(x)=\lambda \psi(x), \quad x \in \mathbb{R} / \pi \mathbb{Z}
$$

where the function $Q(x)$ may generically be complex valued and it is assumed to be of bounded variation and periodic, with the base period $\pi$, i.e., $Q(x+\pi)=Q(x)[1]$. The two fundamental solutions to eq. (1.1) can be cast into the form that reveals their periodicity which is possible due to the Floquet's Theorem (for more details see appendix A). Moreover, the Oscillation Theorem asserts that the (real) spectrum of the Hill's operator with real $Q(x)$ reveals a band structure [1]. The bands are open sets in the positive real line, separated by gaps. The solutions that depend on spectral values that fall into the bands have bounded variation in opposite to those whose spectral values fall into gaps.

The simplest, but very important special cases of the Hill equation are the Mathieu equation [2] which assumes the form:

$$
Q(x)=2 h^{2} \cos 2 x
$$

and the Whittaker-Hill equation [1] for which ${ }^{1}$

$$
Q(x)=\mathrm{A} \cos 4 x+\mathrm{B} \cos 2 x .
$$

\footnotetext{
${ }^{1}$ In the present work: $\mathrm{A}=\frac{1}{2} h^{2}, \mathrm{~B}=4 h \mu$.
} 
Eqs. (1.1)-(1.2) and (1.1)-(1.3) occur in a broad spectrum of physical problems. Their solutions proved useful in many fields of engineering [3, 4], quantum chemistry [5] and pure physics ranging from some topologically non-trivial gauge theories $[6]$ to cosmology $[7,8]$ and D-brane physics [9-11]. Obviously, Schrödinger operators with periodic potentials are of special importance in solid state physics. The potentials (1.2) and (1.3) are real, hence corresponding quantum-mechanical (QM) hamiltonians are hermitian and have real spectra. Recall, that also some complex potentials have applications in quantum physics, for instance, in nuclear theory [12]. Other especially interesting complex potentials are those which yield PT-symmetric QM hamiltonians. ${ }^{2}$ Case studies show that PT-symmetric hamiltonians may have real spectra. ${ }^{3}$ In the present work we consider the eigenvalue problem (1.1) with the complex potential:

$$
Q(x)=\frac{1}{4} h^{2} \mathrm{e}^{-4 i x}+2 h^{2} \cos 2 x
$$

which is obviously PT-symmetric for $h, x \in \mathbb{R}^{4}$

The Mathieu and Whittaker-Hill equations can be studied using conventional, well known methods such as the Hill determinant or WKB, cf. e.g. [1, 18-20]. On the other hand, it has been observed lately that Schrödinger equations with potentials (1.2)-(1.4) emerge entirely within the framework of two-dimensional conformal field theory (2dCFT) as the classical limit of the null vector decoupling (NVD) equations obeyed by certain 3point degenerate irregular conformal blocks [21-25]. Moreover, as a manifestation of the correspondence between the "semiclassical" 2dCFT and the Nekrasov-Shatashvili limit of the $\Omega$-deformed $\mathcal{N}=2$ super Yang-Mills theories (cf. figure 1) the spectrum of the Mathieu and related operators can be investigated with the use of tools of the $\mathcal{N}=2$ SUSY gauge theories, cf. e.g. [22, 26].

Interestingly, it is not the first time when the Mathieu equation appears within the framework of $2 \mathrm{dCFT}$. The (modified) Mathieu equation was also studied by Zamolodchikov in the context of the so-called ODE/IM correspondence [27-29]. ${ }^{5}$ The abbreviation ODE stands for "ordinary differential equations" and IM for "integrable models". The ODE/IM duality also connects 2dCFT to generalized spectral problems which include PT-symmetric Schrödinger eigenvalue problems as well. ${ }^{6}$

\footnotetext{
${ }^{2}$ The hamiltonian $\mathcal{H}$ is $\mathrm{PT}$-symmetric if it satisfies $\mathcal{H}=(\mathrm{PT}) \mathcal{H}(\mathrm{PT})$, where the symbol $\mathrm{P}$ represents the space reflection operator (parity operator) and $\mathrm{T}$ stands for the time reversal operator, cf. [13]. The effect of $\mathrm{P}$ and $\mathrm{T}$ on the $\mathrm{QM}$ coordinate operator $\hat{x}$ and the momentum operator $\hat{p}$ is as follows:

$$
\begin{array}{ll}
\mathrm{P} \hat{x} \mathrm{P}=-\hat{x}, & \mathrm{P} \hat{p} \mathrm{P}=-\hat{p}, \\
\mathrm{~T} \hat{x} \mathrm{~T}=\hat{x}, & \mathrm{~T} \hat{p} \mathrm{~T}=-\hat{p} .
\end{array}
$$

In the Schrödinger eigenvalue problem $\mathcal{H} \psi=\lambda \psi$ we have $\hat{x} \mapsto x$ and $\hat{p} \mapsto-i \frac{d}{d x}$. The parity operator $\mathrm{P}$ is a linear operator and that it leaves invariant the commutation relation $[\hat{x}, \hat{p}]=i \hbar$. The same is true for $\mathrm{T}$ if it is assumed that $\mathrm{T} i \mathrm{~T}=-i$. Since $\mathrm{P}$ and $\mathrm{T}$ are reflection operators, their squares are the unit operator: $\mathrm{P}^{2}=\mathrm{T}^{2}=1$. Finally, it follows that $[\mathrm{P}, \mathrm{T}]=0$.

${ }^{3}$ Precisely, the eigenvalues of a particular PT-symmetric hamiltonian are real if every eigenfunction of a PT-symmetric hamiltonian is also an eigenfunction of the PT operator, cf. [13].

${ }^{4} \mathrm{PT}$-symmetric complex potentials satisfy $\overline{Q(x)}=Q(-x)$.

${ }^{5}$ Cf. the talk by V. Bazhanov [http://cft-im.bo.infn.it/2011/talks/Bazhanov.pdf].

${ }^{6}$ For more details, see the excellent introduction to the subject [29].
} 


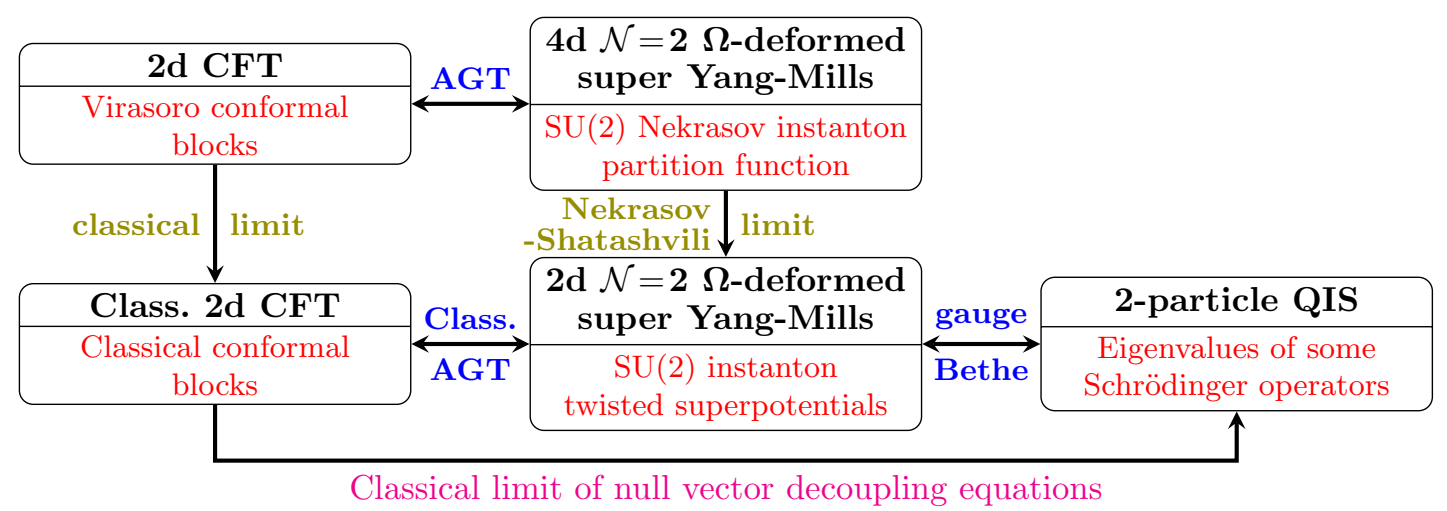

Figure 1. The triple correspondence in the case of the Virasoro classical conformal blocks links the latter to $\mathrm{SU}(2)$ instanton twisted superpotentials which describe the spectra of some quantummechanical systems. The Bethe/gauge correspondence on the r.h.s. connects the $\mathrm{SU}(N) \mathcal{N}=2$ SYM theories with the N-particle quantum integrable systems. An extension of the above triple relation to the case $\mathrm{N}>2$ needs to consider on the l.h.s. the classical limit of the $W_{\mathrm{N}}$ symmetry conformal blocks according to the known extension [14] of the AGT conjecture (see e.g. [15-17]).

In our previous works $[22,23]$ we have found that the Mathieu eigenvalue can be expressed in terms of the pure gauge classical irregular block and such expression exactly coincides with the well known weak coupling expansion of the Mathieu eigenvalue in the case in which the auxiliary parameter is the non-integer Floquet exponent. Furthermore, it has been shown that the formula for the corresponding eigenfunction obtained from the irregular block reproduces the so-called Mathieu exponent from which the non-integer order elliptic cosine and sine functions may be constructed.

In the present paper we continue the line of research initiated in $[22,23]$ and study the eigenvalue problem (1.1) for potentials (1.3) and (1.4) using methods of $2 \mathrm{dCFT}$. The purpose of this work is to answer the question of what kind of solutions are possible to be obtained in this way. This knowledge paves the way for studying spectra of Schrödinger operators with potentials (1.3) and (1.4) employing non-perturbative tools of 2dCFT. Precisely, it seems to be possible to connect different regions of spectra of mentioned operators using duality relations for four-point spherical conformal blocks. This is the main motivation for our research.

The organization of the paper is as follows. In section 2 we introduce the Gaiotto vectors $(\mathrm{GV})$ related to the $\mathcal{N}=2 \mathrm{SYM}$ theories with $N_{f}=0,1$ flavors and define two types of irregular conformal blocks, i.e.: $(a)$ the products of $\mathrm{GV}$, and $(b)$ the matrix elements of certain degenerate chiral vertex operators between GV. Then, we discuss some basic properties of these blocks. In particular, we propose a classical asymptotic behavior for irregular blocks of the type $(a)$ which is inspired by the semiclassical behavior of the physical Liouville field theory correlators and consistent with the Nekrasov-Shatashvili limit of the corresponding Nekrasov instanton functions. Moreover, using this proposal we compute power expansions of the classical $N_{f}=1,2$ irregular conformal blocks. ${ }^{7}$ Finally, we derive

\footnotetext{
${ }^{7} \mathrm{Cf}$. [24, 30], where the classical irregular blocks have been also studied using methods of matrix models.
} 
certain null vector decoupling equations obeyed by the $N_{f}=1,2$ degenerate irregular blocks of the type $(b)$.

In section 3 we derive classical limit of the NVD equations fulfilled by the $N_{f}=1,2$ degenerate irregular blocks. As a result we get closed expressions for some solutions of the eigenvalue problem (1.1) with the PT-symmetric complex potential (1.4) and the Whittaker-Hill potential (1.3). ${ }^{8}$ More concretely, we have found that (i) for each potential (1.3) and (1.4) the corresponding eigenvalue $\lambda$ and the two independent solutions of the eq. (1.1) are given in terms of the classical limit of irregular blocks; (ii) for the complex potential (1.4) the spectrum $\lambda$ is indeed real for $h, x \in \mathbb{R}$ and $\nu \in \mathbb{R} \backslash \mathbb{Z}$; (iii) our fundamental solutions to eqs. (1.1)-(1.3) and (1.1)-(1.4) are nothing but the non-integer order Floquet solutions in the weak coupling (small $h$ ) region. ${ }^{9}$

In section 4 we present our conclusions. The problems that are still open and the possible extensions of the present work are discussed.

\section{Quantum and classical $N_{f}=1,2$ irregular blocks}

As we have mentioned earlier in our previous paper in the sequel [23] the Moore-Seiberg formalism of rational conformal field theory can be successfully extended to the case of non-rational $2 \mathrm{dCFT}$. Therefore the central role in the forthcoming discussion is played by the chiral vertex operators (CVO's) that constitute building blocks for physical fields in the Moore-Seiberg formalism. CVO's are assumed here to act between highest weight representations of Virasoro algebra.

\subsection{Regular and $N_{f}=1,2$ irregular blocks}

For the sake of definiteness let $\mathcal{V}_{c, \Delta}^{n}$ denote the vector space generated by all vectors of the form

$$
\left|\nu_{\Delta, I}\right\rangle=L_{-I}\left|\nu_{\Delta}\right\rangle:=L_{-k_{1}} \ldots L_{-k_{j-1}} L_{-k_{j}}\left|\nu_{\Delta}\right\rangle, \quad \underset{i \in \mathbb{N}}{\forall} L_{-i} \in \operatorname{Vir}_{c}
$$

where $I=\left(k_{1}, \ldots, k_{j-1}, k_{j}\right)$ is an ordered $\left(k_{1} \geq \ldots \geq k_{j} \geq 1\right)$ sequence of positive integers of the length $|I| \equiv k_{1}+\ldots+k_{j}=n$, and $\left|\nu_{\Delta}\right\rangle$ is the highest weight vector:

$$
L_{0}\left|\nu_{\Delta}\right\rangle=\Delta\left|\nu_{\Delta}\right\rangle, \underset{n \in \mathbb{N}}{\forall} L_{n}\left|\nu_{\Delta}\right\rangle=0
$$

The Verma module of the central charge $c$ and the highest weight $\Delta$ is the $\mathbb{Z}$-graded representation of the Virasoro algebra determined on the space:

$$
\mathcal{V}_{c, \Delta}=\bigoplus_{n \geq 0} \mathcal{V}_{c, \Delta}^{n}, \quad \operatorname{dim} \mathcal{V}_{c, \Delta}^{n}=p(n)
$$

\footnotetext{
${ }^{8}$ Note that an appropriate substitution transforms the Whittaker-Hill equation to the so-called equation of Ince, cf. [http://dlmf.nist.gov/28.31]. Hence, our formulae may also be useful to express some solutions of the Ince equation.

${ }^{9}$ For the definition and classification of the Floquet solutions of the Hill equation, see appendix A.
} 
where $p(n)$ is the number of partitions of $n$ (with the convention $p(0)=1$ ). It is an eigenspace of $L_{0}$ with the eigenvalue $\Delta+n$. On $\mathcal{V}_{c, \Delta}^{n}$ there exists the symmetric bilinear form $\langle\cdot \mid \cdot\rangle$ uniquely defined by the relations

$$
\left\langle\nu_{\Delta} \mid \nu_{\Delta}\right\rangle=1 \quad \text { and } \quad\left(L_{n}\right)^{\dagger}=L_{-n}
$$

The Gram matrix $G_{c, \Delta}$ of the form $\langle\cdot \mid \cdot\rangle$ is block-diagonal in the basis $\left\{\left|\nu_{\Delta, I}\right\rangle\right\}$ with blocks

$$
\left[G_{c, \Delta}^{n}\right]_{I J}=\left\langle\nu_{\Delta, I} \mid \nu_{\Delta, J}\right\rangle=\left\langle\nu_{\Delta}\left|\left(L_{-I}\right)^{\dagger} L_{-J}\right| \nu_{\Delta}\right\rangle
$$

The Verma module $\mathcal{V}_{c, \Delta}$ is irreducible if and only if the form $\langle\cdot \mid \cdot\rangle$ is non-degenerate. The criterion for irreducibility is vanishing of the determinant $\operatorname{det} G_{c, \Delta}^{n}$ of the Gram matrix, known as the Kac determinant, given by the formula [31-35]:

$$
\operatorname{det} G_{c, \Delta}^{n}=C_{n} \prod_{1 \leq r s \leq n}\left(\Delta-\Delta_{r s}\right)^{p(n-r s)}
$$

where $C_{n}$ is a constant and $\Delta_{r s}$ are the weights form the Kac table

$$
\Delta_{r s}(c)=\frac{\mathrm{Q}^{2}}{4}-\frac{1}{4}\left(r b+\frac{s}{b}\right)^{2}, \quad r, s \in \mathbb{N},
$$

for which the central charge is given by $c=1+6 \mathrm{Q}^{2}$ with $\mathrm{Q}=b+b^{-1}$.

The non-zero element $\left|\chi_{r s}\right\rangle \in \mathcal{V}_{c, \Delta_{r s}(c)}$ of degree $n=r s$ is called a null vector if $L_{0}\left|\chi_{r s}\right\rangle=\left(\Delta_{r s}+r s\right)\left|\chi_{r s}\right\rangle$, and $L_{k}\left|\chi_{r s}\right\rangle=0, \forall k>0$. Hence, $\left|\chi_{r s}\right\rangle$ is the highest weight state which generates its own Verma module $\mathcal{V}_{c, \Delta_{r s}(c)+r s}$, which is a submodule of $\mathcal{V}_{c, \Delta_{r s}(c)}$. One can prove that each submodule of the Verma module $\mathcal{V}_{c, \Delta_{r s}(c)}$ is generated by a null vector. Then, the module $\mathcal{V}_{c, \Delta_{r s}(c)}$ is irreducible if and only if it does not contain null vectors with positive degree.

For non-degenerate values of $\Delta$, i.e. for $\Delta \neq \Delta_{r s}(c)$, there exists the basis $\left\{\left|\nu_{\Delta, I}^{t}\right\rangle\right\}_{I \vdash n}$ in $\mathcal{V}_{c, \Delta}^{n}$ whose elements are defined by the relation $\left\langle\nu_{\Delta, I}^{t} \mid \nu_{\Delta, J}\right\rangle=\delta_{I J}$ for all $\left|\nu_{\Delta, J}\right\rangle \in \mathcal{V}_{c, \Delta}^{n}$. The basis vectors $\left|\nu_{\Delta, I}^{t}\right\rangle$ have the following representation in the standard basis

$$
\left|\nu_{\Delta, I}^{t}\right\rangle:=\sum_{J \vdash n}\left[G_{c, \Delta}^{n}\right]^{I J}\left|\nu_{\Delta, J}\right\rangle, \quad \sum_{K \vdash n}\left[G_{c, \Delta}^{n}\right]^{I K}\left[G_{c, \Delta}^{n}\right]_{K J}=\delta_{J}^{I} .
$$

The chiral vertex operator is the linear map $V_{\infty}^{\Delta_{3} \Delta_{2} \Delta_{1}}: \mathcal{V}_{\Delta_{2}} \otimes \mathcal{V}_{\Delta_{1}} \rightarrow \mathcal{V}_{\Delta_{3}}$ such that for all $\left|\xi_{2}\right\rangle \in \mathcal{V}_{\Delta_{2}}$ the operator

$$
V\left(\xi_{2} \mid z\right) \equiv V_{\infty}^{\Delta_{3} \Delta_{2} \Delta_{1}}\left(\left|\xi_{2}\right\rangle \otimes \cdot\right): \mathcal{V}_{\Delta_{1}} \rightarrow \mathcal{V}_{\Delta_{3}}
$$

satisfies the following conditions

$$
\begin{aligned}
{\left[L_{n}, V\left(\nu_{2} \mid z\right)\right] } & =z^{n}\left(z \frac{\partial}{\partial z}+(n+1) \Delta_{2}\right) V\left(\nu_{2} \mid z\right), & n \in \mathbb{Z} \\
V\left(L_{-1} \xi_{2} \mid z\right) & =\frac{\partial}{\partial z} V\left(\xi_{2} \mid z\right) &
\end{aligned}
$$




$$
\begin{array}{rlr}
V\left(L_{n} \xi_{2} \mid z\right)= & \sum_{k=0}^{n+1}\left(\begin{array}{c}
n+1 \\
k
\end{array}\right)(-z)^{k}\left[L_{n-k}, V\left(\xi_{2} \mid z\right)\right], & n>-1, \\
V\left(L_{-n} \xi_{2} \mid z\right)= & \sum_{k=0}^{\infty}\left(\begin{array}{c}
n-2+k \\
n-2
\end{array}\right) z^{k} L_{-n-k} V\left(\xi_{2} \mid z\right) & \\
& +(-1)^{n} \sum_{k=0}^{\infty}\left(\begin{array}{c}
n-2+k \\
n-2
\end{array}\right) z^{-n+1-k} V\left(\xi_{2} \mid z\right) L_{k-1}, & n>1 .
\end{array}
$$

The commutation relation (2.5) defines the primary vertex operator corresponding to the highest weight state $\left|\nu_{2}\right\rangle \in \mathcal{V}_{\Delta_{2}}$. The matrix element of the primary CVO between basis states in $\mathcal{V}_{\Delta_{a, c}}^{|I|,|J|}$ fulfills the following relation

$$
\left\langle\nu_{\Delta_{a}, I}\left|V\left(\nu_{\Delta_{b}} \mid z\right)\right| \nu_{\Delta_{c}, J}\right\rangle=z^{\Delta_{a}-\Delta_{b}-\Delta_{c}+|I|-|J|}\left\langle\nu_{\Delta_{a}}\left|V\left(\nu_{\Delta_{b}} \mid 1\right)\right| \nu_{\Delta_{c}}\right\rangle .
$$

In what follows we assume the normalization $\left\langle\nu_{\Delta_{a}}\left|V\left(\nu_{\Delta_{b}} \mid 1\right)\right| \nu_{\Delta_{c}}\right\rangle=1$.

In analogy to the Heisenberg algebra it is possible to form coherent states for generators of the Virasoro algebra. Indeed, as it was first shown by Gaiotto [36] and in refs. [25, 37-39] the Virasoro generators for each $n>0$ in the highest weight condition in eq. (2.2) may be treated as "annihilation" operators. Hence, there is a vector on which a certain finite set of positive indexed generators of Virasoro algebra act diagonally termed the irregular vector. The Virasoro algebra each positive indexed generator obeys induces corresponding algebra of differential operators in the space of parameters labeling the irregular vector [39]. In what follows we are concerned with only two simplest cases that due to AGT correspond to gauge theories with $N_{f}=0$ and $N_{f}=1$, where there are at most two parameters labeling the irregular vector. ${ }^{10}$

Let us recall that the Virasoro irregular vector that is a "coherent state" of $L_{1}$ to which we further refer as to the "zero flavor" or $N_{f}=0$ state fulfills the following conditions:

$$
L_{0}\left|\Delta, \Lambda^{2}\right\rangle=\left(\Delta+\frac{\Lambda}{2} \frac{\partial}{\partial \Lambda}\right)\left|\Delta, \Lambda^{2}\right\rangle, \quad L_{1}\left|\Delta, \Lambda^{2}\right\rangle=\Lambda^{2}\left|\Delta, \Lambda^{2}\right\rangle, \quad L_{n}\left|\Delta, \Lambda^{2}\right\rangle=0 \quad \forall n \geq 2 .
$$

Making use of the above algebra it can be developed in $\mathcal{V}_{c, \Delta}$ as follows ${ }^{11}$

$$
\left|\Delta, \Lambda^{2}\right\rangle=\sum_{I \in \mathbb{Y}}\left|\nu_{\Delta, I}\right\rangle\left\langle\nu_{\Delta, I}^{t} \mid \Delta, \Lambda^{2}\right\rangle=\sum_{n=0}^{\infty} \Lambda^{2 n} \sum_{I \vdash n}\left[G_{c, \Delta}^{n}\right]^{\left(1^{n}\right) I}\left|\nu_{\Delta, I}\right\rangle,
$$

where we used the projector (identity operator in $\mathcal{V}_{c, \Delta}$ ):

$$
\mathbb{P}_{\Delta}=\sum_{I \in \mathbb{Y}}\left|\nu_{\Delta, I}\right\rangle \otimes\left\langle\nu_{\Delta, I}^{t}\left|=\sum_{n \geq 0} \sum_{I, J \vdash n}\left[G_{c, \Delta}^{n}\right]^{I J}\right| \nu_{\Delta, I}\right\rangle \otimes\left\langle\nu_{\Delta, J}\right|,
$$

\footnotetext{
${ }^{10}$ In the present paper we adopt the following nomenclature: "zero flavor", "single flavor" and so on for Gaiotto's states and then for irregular blocks as these objects are related to corresponding quantities in the $\mathcal{N}=2$ super-Yang-Mills theories with $N_{f}=0,1, \ldots$ - zero, one and more flavors.

${ }^{11}$ The symbol $\mathbb{Y}$ denotes the set of all partitions or equivalently the Young diagrams of all natural numbers that in the mathematical literature is termed the Young lattice.
} 
and a convenient normalization $\left\langle\nu_{\Delta} \mid \Delta, \Lambda^{2}\right\rangle=1$. The irregular vector to which we further refer as to the "single flavor" or $N_{f}=1$ state fulfills the following defining conditions

$$
\begin{array}{ll}
L_{0}|\Delta, \Lambda, m\rangle=\left(\Delta+\Lambda \frac{\partial}{\partial \Lambda}\right)|\Delta, \Lambda, m\rangle, & L_{1}|\Delta, \Lambda, m\rangle=m \Lambda|\Delta, \Lambda, m\rangle, \\
L_{2}|\Delta, \Lambda, m\rangle=\Lambda^{2}|\Delta, \Lambda, m\rangle, & L_{n}|\Delta, \Lambda, m\rangle=0 \quad \forall n \geq 3 .
\end{array}
$$

Proceeding analogously as in $N_{f}=0$ case we obtain

$$
|\Delta, \Lambda, m\rangle=\sum_{n=0}^{\infty} \Lambda^{n} \sum_{p=0}^{\left[\frac{n}{2}\right]} m^{n-2 p} \sum_{I \vdash n}\left[G_{c, \Delta}^{n}\right]^{\left(1^{n-2 p} 2^{p}\right) I}\left|\nu_{\Delta, I}^{n}\right\rangle .
$$

The quantum irregular conformal blocks are defined as inner products of the irregular vectors $[36,37]:{ }^{12}$

$$
\begin{aligned}
\mathcal{F}_{c, \Delta}(\Lambda, m) & =\left\langle\Delta, \frac{1}{2} \Lambda, 2 m \mid \Delta, \Lambda^{2}\right\rangle \\
& =\sum_{n=0}^{\infty}\left(\frac{1}{2} \Lambda^{3}\right)^{n} \sum_{p=0}^{\left[\frac{n}{2}\right]}(2 m)^{n-2 p}\left[G_{c, \Delta}^{n}\right]^{\left(1^{n-2 p} 2^{p}\right)\left(1^{n}\right)} \\
\mathcal{F}_{c, \Delta}\left(\Lambda, m_{1}, m_{2}\right) & =\left\langle\Delta, \frac{1}{2} \Lambda, 2 m_{1} \mid \Delta, \frac{1}{2} \Lambda, 2 m_{2}\right\rangle \\
& =\sum_{n=0}^{\infty}\left(\frac{\Lambda}{2}\right)^{2 n} \sum_{p, p^{\prime}=0}^{\left[\frac{n}{2}\right]}\left(2 m_{1}\right)^{n-2 p}\left[G_{c, \Delta}^{n}\right]^{\left(1^{n-2 p} 2^{p}\right)\left(1^{n-2 p} 2^{p}\right)}\left(2 m_{2}\right)^{n-2 p^{\prime}}
\end{aligned}
$$

Irregular blocks (2.11)-(2.12) can be recovered from conformal blocks on the torus and on the sphere in a properly defined decoupling limits of external conformal weights [37, 40]. To see this let $C_{g, n}$ denote the Riemann surface with genus $g$ and $n$ punctures. Let $x$ be the modular parameter of the 4-punctured Riemann sphere then the $s$-channel conformal block on $C_{0,4}$ is defined as the following formal $x$-expansion:

$$
\mathcal{F}_{c, \Delta}\left[\begin{array}{cc}
\Delta_{2} & \Delta_{3} \\
\Delta_{1} & \Delta_{4}
\end{array}\right](x)=x^{\Delta-\Delta_{3}-\Delta_{4}}\left(1+\sum_{n=1}^{\infty} x^{n} \mathcal{F}_{c, \Delta}^{n}\left[\begin{array}{cc}
\Delta_{2} & \Delta_{3} \\
\Delta_{1} & \Delta_{4}
\end{array}\right]\right),
$$

where the coefficients of the conformal block are defined as

$$
\mathcal{F}_{c, \Delta}^{n}\left[\begin{array}{cc}
\Delta_{2} & \Delta_{3} \\
\Delta_{1} & \Delta_{4}
\end{array}\right]=\sum_{I, J \vdash n}\left\langle\nu_{\Delta_{1}}\left|V_{\Delta_{2}}(1)\right| \nu_{\Delta, I}^{n}\right\rangle\left[G_{c, \Delta}^{n}\right]^{I J}\left\langle\nu_{\Delta, J}^{n}\left|V_{\Delta_{3}}(1)\right| \nu_{\Delta_{4}}\right\rangle .
$$

\footnotetext{
${ }^{12}$ In fact, there is much more Gaiotto's states than just these two written in eqs. (2.10G1) and (2.10G2), see for instance $[25,38,39]$. In the present paper we confine ourselves to study irregular blocks being inner products of (2.10G1), (2.10G2) or matrix elements between these two states. Possible extensions of the present work taking into account existence of the other Gaiotto states are discussed in conclusions.
} 
Now, employing a suitable AGT inspired parametrization of the external weights $\Delta_{i}$ and the central charge $c$, i.e.:

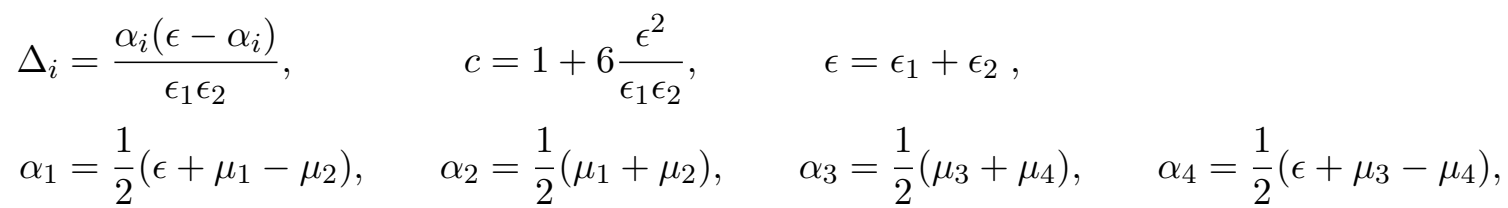

and introducing the dimensionless expansion parameters: $\Lambda=\hat{\Lambda} /\left(-\epsilon_{1} \epsilon_{2}\right)^{\frac{1}{2}}$ and $m_{i}=$ $\hat{m}_{i} /\left(-\epsilon_{1} \epsilon_{2}\right)^{\frac{1}{2}}$, where $\hat{m}=\mu_{1}-\frac{1}{2} \epsilon, \hat{m}_{1,4}=\mu_{1,4}-\frac{1}{2} \epsilon$, one obtains [37, 40]:

$$
\begin{aligned}
& x^{\Delta_{3}+\Delta_{4}-\Delta} \mathcal{F}_{c, \Delta}\left[\begin{array}{cc}
\Delta_{2} & \Delta_{3} \\
\Delta_{1} & \Delta_{4}
\end{array}\right](x) \underset{x \mu_{2} \mu_{3} \mu_{4}=\hat{\Lambda}^{3}}{\stackrel{\mu_{2}, \mu_{3}, \mu_{4} \rightarrow \infty}{\longrightarrow}} \mathcal{F}_{c, \Delta}(\Lambda, m), \\
& x^{\Delta_{3}+\Delta_{4}-\Delta} \mathcal{F}_{c, \Delta}\left[\begin{array}{cc}
\Delta_{2} & \Delta_{3} \\
\Delta_{1} & \Delta_{4}
\end{array}\right](x) \underset{x \mu_{2} \mu_{3}=\hat{\Lambda}^{2}}{\stackrel{\mu_{2}, \mu_{3} \rightarrow \infty}{\longrightarrow}} \mathcal{F}_{c, \Delta}\left(\Lambda, m_{1}, m_{4}\right) \text {. }
\end{aligned}
$$

The above conformal blocks may be related by means of AGT correspondence to their gauge theoretic counterparts, that is $\mathrm{SU}(2)$ Nekrasov's instanton partition functions with $N_{f}=1,2$ flavors [36, 41-43]:

$$
\begin{aligned}
\mathcal{F}_{c, \Delta}(\Lambda, m) & =\mathcal{Z}_{\text {inst }}^{\mathrm{SU}(2), N_{f}=1}\left(\hat{\Lambda}, a, \hat{m}, \epsilon_{1}, \epsilon_{2}\right), \\
\mathcal{F}_{c, \Delta}\left(\Lambda, m_{1}, m_{2}\right) & =\mathcal{Z}_{\text {inst }}^{\mathrm{SU}(2), N_{f}=2}\left(\hat{\Lambda}, a, \hat{m}_{1}, \hat{m}_{2}, \epsilon_{1}, \epsilon_{2}\right) .
\end{aligned}
$$

The relations (2.14)-(2.15), which are understood as equalities between the coefficients of the expansions of both sides, hold for

$$
\Lambda=\frac{\hat{\Lambda}}{\sqrt{-\epsilon_{1} \epsilon_{2}}}, \quad m_{i}=\frac{\hat{m}_{i}}{\sqrt{-\epsilon_{1} \epsilon_{2}}}, \quad \Delta=\frac{\epsilon^{2}-4 a^{2}}{4 \epsilon_{1} \epsilon_{2}}, \quad c=1+6 \frac{\epsilon^{2}}{\epsilon_{1} \epsilon_{2}} \equiv 1+6 \mathrm{Q}^{2}
$$

where

$$
\mathrm{Q}=b+\frac{1}{b} \equiv \sqrt{\frac{\epsilon_{2}}{\epsilon_{1}}}+\sqrt{\frac{\epsilon_{1}}{\epsilon_{2}}}, \quad b=\sqrt{\frac{\epsilon_{2}}{\epsilon_{1}}} .
$$

The study of the Nekrasov partition functions $\mathcal{Z}_{\text {Nekrasov }}=\mathcal{Z}_{\text {pert }} \mathcal{Z}_{\text {inst }}$ in the limit $\epsilon_{2} \rightarrow 0$ has revealed that it behaves exponentially [44]. In particular, for the instanton part of the partition function we have

$$
\mathcal{Z}_{\text {inst }}\left(\cdot, \epsilon_{1}, \epsilon_{2}\right) \stackrel{\epsilon_{2} \rightarrow 0}{\sim} \exp \left\{\frac{1}{\epsilon_{2}} \mathcal{W}_{\text {inst }}\left(\cdot, \epsilon_{1}\right)\right\} .
$$

Therefore, in view of eqs. (2.14)-(2.15), and relation between Liouville and deformation parameters in eq. (2.17) from the Nekrasov-Shatashvili limit in eq. (2.18) one can expect the exponential behavior of irregular blocks in the limit $b \rightarrow 0$, i.e.:

$$
\begin{gathered}
\mathcal{F}_{1+6 Q^{2}, \Delta}(\Lambda, m) \stackrel{b \rightarrow 0}{\sim} \exp \left\{\frac{1}{b^{2}} f_{\delta}^{\mathbf{1}}\left(\hat{\Lambda} / \epsilon_{1}, \hat{m} / \epsilon_{1}\right)\right\}, \\
\mathcal{F}_{1+6 Q^{2}, \Delta}\left(\Lambda, m_{1}, m_{2}\right) \stackrel{b \rightarrow 0}{\sim} \exp \left\{\frac{1}{b^{2}} f_{\delta}^{\mathbf{2}}\left(\hat{\Lambda} / \epsilon_{1}, \hat{m}_{1} / \epsilon_{1}, \hat{m}_{2} / \epsilon_{1}\right)\right\},
\end{gathered}
$$


where $\Delta=\frac{1}{b^{2}} \delta, \delta=\mathcal{O}\left(b^{0}\right)$. The classical behaviors (2.19)-(2.20) are very nontrivial statements concerning quantum irregular blocks. Although there is no proof of eqs. (2.19)-(2.20) the existence of the classical irregular blocks $f^{1}, f^{2}$ can be verified through direct calculation order by order in $\hat{\Lambda} / \epsilon_{1}$. Using power expansions of quantum irregular blocks (2.11)(2.12) and eqs. (2.19) $-(2.20)$ up to $n=3$ one finds

(i) the classical irregular block with "single flavor" $N_{f}=1$ :

$$
\begin{aligned}
f_{\delta}^{\mathbf{1}}\left(\hat{\Lambda} / \epsilon_{1}, \hat{m} / \epsilon_{1}\right) & =\lim _{b \rightarrow 0} b^{2} \log \mathcal{F}_{1+6 Q^{2}, \frac{1}{b^{2}} \delta}\left(\hat{\Lambda} /\left(\epsilon_{1} b\right), \hat{m} /\left(\epsilon_{1} b\right)\right) \\
& =\sum_{n=1}^{\infty}\left(\hat{\Lambda} / \epsilon_{1}\right)^{3 n} f_{\delta}^{\mathbf{1}, n}\left(\frac{\hat{m}}{\epsilon_{1}}\right),
\end{aligned}
$$

where the coefficients read as follows

$$
\begin{aligned}
f_{\delta}^{\mathbf{1}, 1}\left(\frac{\hat{m}}{\epsilon_{1}}\right) & =\frac{1}{2 \delta} \frac{\hat{m}}{\epsilon_{1}} \\
f_{\delta}^{\mathbf{1}, 2}\left(\frac{\hat{m}}{\epsilon_{1}}\right) & =\frac{5 \delta-3}{16 \delta^{3}(4 \delta+3)}\left(\frac{\hat{m}}{\epsilon_{1}}\right)^{2}-\frac{3}{16 \delta(4 \delta+3)}, \\
f_{\delta}^{\mathbf{1}, 3}\left(\frac{\hat{m}}{\epsilon_{1}}\right) & =\frac{\delta(9 \delta-19)+6}{48 \delta^{5}(\delta+2)(4 \delta+3)}\left(\frac{\hat{m}}{\epsilon_{1}}\right)^{3}+\frac{6-7 \delta}{48 \delta^{3}(\delta+2)(4 \delta+3)} \frac{\hat{m}}{\epsilon_{1}} ;
\end{aligned}
$$

(ii) the classical irregular block with "two flavors" $N_{f}=2$ :

$$
\begin{aligned}
f_{\delta}^{\mathbf{2}}\left(\hat{\Lambda} / \epsilon_{1}, \hat{m}_{1} / \epsilon_{1}, \hat{m}_{2} / \epsilon_{1}\right) & =\lim _{b \rightarrow 0} b^{2} \log \mathcal{F}_{1+6 Q^{2}, \frac{1}{b^{2}} \delta}\left(\hat{\Lambda} /\left(\epsilon_{1} b\right), \hat{m}_{2} /\left(\epsilon_{1} b\right), \hat{m}_{1} /\left(\epsilon_{1} b\right)\right) \\
& =\sum_{n=1}^{\infty}\left(\hat{\Lambda} / \epsilon_{1}\right)^{2 n} f_{\delta}^{\mathbf{2}, n}\left(\frac{\hat{m}_{1}}{\epsilon_{1}}, \frac{\hat{m}_{2}}{\epsilon_{1}}\right),
\end{aligned}
$$

where the coefficients are of the form

$$
\begin{aligned}
f_{\delta}^{2,1}\left(\frac{\hat{m}_{1}}{\epsilon_{1}}, \frac{\hat{m}_{2}}{\epsilon_{1}}\right)= & \frac{1}{2 \delta} \frac{\hat{m}_{1}}{\epsilon_{1}} \frac{\hat{m}_{2}}{\epsilon_{1}}, \\
f_{\delta}^{\mathbf{2}, 2}\left(\frac{\hat{m}_{1}}{\epsilon_{1}}, \frac{\hat{m}_{2}}{\epsilon_{1}}\right)= & \frac{\delta^{2}\left(\delta-3\left(\frac{\hat{m}_{2}}{\epsilon_{1}}\right)^{2}\right)+\left(\frac{\hat{m}_{1}}{\epsilon_{1}}\right)^{2}\left((5 \delta-3)\left(\frac{\hat{m}_{2}}{\epsilon_{1}}\right)^{2}-3 \delta^{2}\right)}{16 \delta^{3}(4 \delta+3)}, \\
f_{\delta}^{\mathbf{2}, 3}\left(\frac{\hat{m}_{1}}{\epsilon_{1}}, \frac{\hat{m}_{2}}{\epsilon_{1}}\right)= & \frac{1}{48 \delta^{5}(\delta+2)(4 \delta+3)} \frac{\hat{m}_{1}}{\epsilon_{1}} \frac{\hat{m}_{2}}{\epsilon_{1}}\left[(6-7 \delta) \delta^{2}\left(\frac{\hat{m}_{2}}{\epsilon_{1}}\right)^{2}\right. \\
& \left.+\left(\frac{\hat{m}_{1}}{\epsilon_{1}}\right)^{2}\left((\delta(9 \delta-19)+6)\left(\frac{\hat{m}_{2}}{\epsilon_{1}}\right)^{2}+(6-7 \delta) \delta^{2}\right)+5 \delta^{4}\right] .
\end{aligned}
$$

As another yet consistency check of our approach let us observe that combining (2.14)(2.17) and (2.19)-(2.20) one gets an identification between classical irregular blocks and $\mathrm{SU}(2) N_{f}=1,2$ effective twisted superpotentials:

$$
f_{\frac{1}{4}-\frac{a^{2}}{\epsilon_{1}^{2}}}^{\mathbf{1 , 2}}\left(\hat{\Lambda} / \epsilon_{1}, \cdot\right)=\frac{1}{\epsilon_{1}} \mathcal{W}_{\text {inst }}^{\mathrm{SU}(2), N_{f}=1,2}\left(\hat{\Lambda}, a, \cdot, \epsilon_{1}\right) .
$$


Note that the classical conformal weight $\delta$ in the eq. (2.23) above is expressed in terms of the gauge theory parameters $a, \epsilon_{1}$. Indeed, one finds

$$
\delta=\lim _{b \rightarrow 0} b^{2} \Delta=\lim _{\epsilon_{2} \rightarrow 0} \frac{\epsilon_{2}}{\epsilon_{1}} \Delta=\frac{1}{4}-\frac{a^{2}}{\epsilon_{1}^{2}} .
$$

By means of the expansions (2.21a)-(2.22a) and theirs analogues for the twisted superpotentials obtained independently from the instanton partition functions one can confirm the identities (2.23) up to desired order.

\section{$2.2 \quad$ Null vector decoupling equations}

In this subsection we shall derive partial differential equations obeyed by the degenerate irregular blocks, cf. [21]. The latter we define as matrix elements of the degenerate chiral vertex operator, ${ }^{13} V_{+}(z)=V\left(\left|\nu_{\Delta_{+}}\right\rangle \mid z\right)$, where $\Delta_{+} \equiv \Delta_{21}=-\frac{3}{4} b^{2}-\frac{1}{2}$, between Gaitto's states (2.10G1)-(2.10G2):

$$
\begin{aligned}
\Psi^{\mathbf{1}}(z ; \Lambda, m) & :=\left\langle\Delta^{\prime}, \frac{1}{2} \Lambda, 2 m\left|V_{+}(z)\right| \tilde{\Delta}, \Lambda^{2}\right\rangle, \\
\Psi^{\mathbf{2}}\left(z ; \Lambda, m_{1}, m_{2}\right) & :=\left\langle\Delta^{\prime}, \frac{1}{2} \Lambda, 2 m_{1}\left|V_{+}(z)\right| \tilde{\Delta}, \frac{1}{2} \Lambda, 2 m_{2}\right\rangle .
\end{aligned}
$$

One of the tools that allows to derive the so-called null vector decoupling (NVD) equations is the following:

Theorem 1 (Feigin-Fuchs [33], cf. [45]). Let $i, j, k \in\{1,2,3\}$ be chosen such that $j \neq i$, $k \neq i, j \neq k$. Let us assume that

1. $\Delta_{i}=\Delta_{r s} \equiv \frac{1}{4} \mathrm{Q}^{2}-\frac{1}{4}\left(r b+s b^{-1}\right)^{2}, r, s \in \mathbb{N}$;

2. the vector $\left|\xi_{i}\right\rangle$ lies in the singular submodule generated by the null vector $\left|\chi_{r s}\right\rangle$, i.e.: $\left|\xi_{i}\right\rangle \in \mathcal{V}_{c, \Delta_{r s}(c)+r s} \subset \mathcal{V}_{c, \Delta_{r s}(c)}$.

Then, $\left\langle\xi_{3}\left|V\left(\xi_{2} \mid z_{2}\right)\right| \xi_{1}\right\rangle=0$ if and only if

$$
\Delta_{j}=\Delta_{\beta_{j}} \equiv \frac{\mathrm{Q}^{2}}{4}-\frac{1}{4} \beta_{j}^{2} \quad \text { and } \quad \Delta_{k}=\Delta_{\beta_{k}} \equiv \frac{\mathrm{Q}^{2}}{4}-\frac{1}{4} \beta_{k}^{2}
$$

satisfy the fusion rules $\beta_{j}-\beta_{k}=p b+q b^{-1}$, where $p \in\{1-r, 3-r, \ldots, r-1\}$ and $q \in\{1-s, 3-s, \ldots, s-1\}$.

We will apply the Feigin-Fuchs Theorem in the case when $\Delta_{2}=\Delta_{+} \equiv \Delta_{21}$. Therefore, we have to assume that the weights $\Delta_{1}=\tilde{\Delta}$ and $\Delta_{3}=\Delta^{\prime}$ of in and out states are related by the fusion rule I or II:

$$
\begin{array}{cllll}
\text { I : } & \tilde{\Delta}=\Delta_{\beta_{1}}, & \Delta^{\prime}=\Delta_{\beta_{3}}=\Delta_{\beta_{1}+b} & \Leftrightarrow & \beta_{3}=\beta_{1}+b, \\
\text { II : } & \tilde{\Delta}=\Delta_{\beta_{1}}, & \Delta^{\prime}=\Delta_{\beta_{3}}=\Delta_{\beta_{1}-b} & \Leftrightarrow & \beta_{3}=\beta_{1}-b .
\end{array}
$$

\footnotetext{
${ }^{13}$ Calculations presented in this subsection hold also for $V_{\Delta_{12}}(z)$.
} 
In our calculation we will use a little bit modified (compering to (2.25)) parametrization of conformal weights, namely

$$
\Delta(\sigma) \equiv \frac{\mathrm{Q}^{2}}{4}-\sigma^{2}
$$

in which the fusion rules assumed above reads as follows

$$
\begin{array}{lll}
\text { I : } & \tilde{\Delta}=\Delta\left(\sigma-\frac{b}{4}\right), & \Delta^{\prime}=\Delta\left(\sigma+\frac{b}{4}\right), \\
\text { II : } & \tilde{\Delta}=\Delta\left(\sigma+\frac{b}{4}\right), & \Delta^{\prime}=\Delta\left(\sigma-\frac{b}{4}\right) .
\end{array}
$$

The matrix elements such as $(2.24 \Psi 1)$ or $(2.24 \Psi 2)$, i.e. with the conformal weights fulfilling (2.26), (2.27) or (2.29), (2.30) will be denoted by $\Psi_{\mathrm{I}}^{\mathrm{i}}, \Psi_{\mathrm{II}}^{\mathrm{i}}$.

Hence, by virtue of the Feigin-Fuchs Theorem we have four equations for $N_{f}=1,2$ written in a concise form as

$$
\left\langle\Delta^{\prime}, \frac{1}{2} \Lambda, 2 m\left|\chi_{+}(z)\right| \tilde{\Delta}, \cdot\right\rangle=0
$$

where

(a) $\chi_{+}(z)$ is the null vertex operator,

$$
\chi_{+}(z)=\left(\widehat{L}_{-2}(z)-\frac{3}{2\left(2 \Delta_{+}+1\right)} \widehat{L}_{-1}^{2}(z)\right) V_{+}(z) \equiv V\left(\left(L_{-2}+\frac{1}{b^{2}} L_{-1}^{2}\right)\left|\nu_{\Delta_{+}}\right\rangle \mid z\right),
$$

which corresponds to the null vector

$$
\left|\chi_{+}\right\rangle=\chi_{+}(0)|0\rangle=\left(L_{-2}+\frac{1}{b^{2}} L_{-1}^{2}\right)\left|\nu_{\Delta_{+}}\right\rangle,
$$

from the second level of the Verma module $\mathcal{V}_{\Delta_{+}}$;

(b) the dot stands for the set of parameters $\Lambda^{2}$ and $\frac{1}{2} \Lambda, 2 m$ for "zero flavor" and "single flavor" irregular vector, respectively;

(c) the conformal weights obey (2.29) or (2.30).

In order to convert eqs. (2.31) to PDE's obeyed by the degenerate irregular blocks $\Psi_{\iota}^{\mathbf{i}}$, $\mathbf{i}=\mathbf{1}, \mathbf{2}, \iota=$ I, II one needs to employ the following Ward identities

$$
\left\langle\Delta^{\prime}, \frac{1}{2} \Lambda, 2 m\left|T(w) V_{+}(z)\right| \tilde{\Delta}, \cdot\right\rangle=\left[\frac{z}{w(w-z)} \frac{\partial}{\partial z}+\frac{\Delta_{+}}{(w-z)^{2}}+U_{\iota}^{\mathrm{i}}\right] \Psi_{\iota}^{\mathrm{i}},
$$

where $^{14}$

$$
\begin{aligned}
& U_{\iota}^{\mathbf{1}}=\frac{1}{4} \Lambda^{2}+\frac{m \Lambda}{w}+\frac{\Lambda^{2}}{w^{3}}+\frac{1}{3 w^{2}}\left(\Lambda \frac{\partial}{\partial \Lambda}+\Delta^{\prime}+2 \tilde{\Delta}-\Delta_{+}-z \frac{\partial}{\partial z}\right) \\
& U_{\iota}^{\mathbf{2}}=\frac{1}{4} \Lambda^{2}+\frac{m_{1} \Lambda}{w}+\frac{m_{2} \Lambda}{w^{3}}+\frac{\frac{1}{4} \Lambda^{2}}{w^{4}}+\frac{1}{2 w^{2}}\left(\Lambda \frac{\partial}{\partial \Lambda}+\Delta^{\prime}+\tilde{\Delta}-\Delta_{+}-z \frac{\partial}{\partial z}\right) .
\end{aligned}
$$

\footnotetext{
${ }^{14}$ Here, $U_{\iota}^{\mathbf{i}}, \mathbf{i}=\mathbf{1}, \mathbf{2}$ depend on $\iota=$ I, II via terms $\Delta^{\prime}+2 \tilde{\Delta}-\Delta_{+}$and $\Delta^{\prime}+\tilde{\Delta}-\Delta_{+}$.
} 
From eq. (2.33) and using the formula [46]:

$$
\widehat{L}_{-k}(z)=\frac{1}{2 \pi i} \oint_{C_{z}} d w(w-z)^{1-k} T(w),
$$

one finds that

$$
\left\langle\Delta^{\prime}, \frac{1}{2} \Lambda, 2 m\left|\hat{L}_{-2}(z) V_{+}(z)\right| \tilde{\Delta}, \cdot\right\rangle=\left[-\frac{1}{z} \frac{\partial}{\partial z}+\left.U_{\iota}^{\mathbf{i}}\right|_{w \rightarrow z}\right] \Psi_{\iota}^{\mathbf{i}}
$$

where $U_{\iota}^{\mathbf{i}}$ are given in eqs. (2.33U1) and (2.33U2). Finally, taking into account that matrix elements of the descendant operator $\widehat{L}_{-1}^{2}(z) V_{+}(z)$ between irregular vectors result in $\partial_{z}^{2} \Psi_{\iota}^{\mathbf{i}}$, from eq. (2.32), eqs. (2.31) and eqs. (2.34) we get the sought-after partial differential equations:

1. for "single flavor" degenerate irregular blocks:

$$
\left[\frac{1}{b^{2}} z^{2} \frac{\partial^{2}}{\partial z^{2}}-\frac{4 z}{3} \frac{\partial}{\partial z}+\frac{1}{4} z^{2} \Lambda^{2}+z m \Lambda+\frac{\Lambda^{2}}{z}+\frac{\Lambda}{3} \frac{\partial}{\partial \Lambda}+\frac{2 \tilde{\Delta}+\Delta^{\prime}-\Delta_{+}}{3}\right] \Psi_{\iota}^{1}=0
$$

2. for "two flavors" degenerate irregular blocks:

$$
\begin{aligned}
{\left[\frac{1}{b^{2}} z^{2} \frac{\partial^{2}}{\partial z^{2}}-\frac{3 z}{2} \frac{\partial}{\partial z}+\frac{1}{4} \Lambda^{2}\right.} & \left(z^{2}+\frac{1}{z^{2}}\right)+\Lambda\left(z m_{1}+\frac{m_{2}}{z}\right) \\
+ & \left.\frac{\Lambda}{2} \frac{\partial}{\partial \Lambda}+\frac{\tilde{\Delta}+\Delta^{\prime}-\Delta_{+}}{2}\right] \Psi_{\iota}^{2}=0 .
\end{aligned}
$$

In the next section we will consider the limit $b \rightarrow 0$ each of these equations separately. The steps in forthcoming analysis follows directly those that has been already done in our previous work [23]. The experience gained in this study helps us to compute the semi-classical limit of eqs. (2.35D1) and (2.35D2).

\section{Classical limit of $N_{f}=1,2$ NVD equations}

The differential equations we derived in the previous section are a starting point to the derivation of the differential equations some of which are well known in mathematics and physics. In what follows we take the classical limit of NVD eqs. (2.35D1) and (2.35D2) as well as the functions that solve them. As steps leading to the equations are the same in both cases $N_{f}=1,2$ we discuss them in full generality.

The functions ${ }^{15} \Psi_{\iota}^{\mathbf{1}}, \Psi_{\iota}^{\mathbf{2}}, \iota=\mathrm{I}$, II that solve eqs. (2.35D1), (2.35D2), that are the degenerate irregular blocks given in eqs. $(2.24 \Psi 1)$ and $(2.24 \Psi 2)$ can be given explicit form

\footnotetext{
${ }^{15}$ Let us recall that indices I, II mean that different fusion rules, namely (2.29) and (2.30), have been assumed.
} 
by means of eqs. (2.10G1), (2.10G2) and eq. (2.9) which results in

$$
\begin{aligned}
\Psi_{\iota}^{\mathbf{1}}(z ; \Lambda, m)= & z^{\Delta^{\prime}-\Delta_{+}-\tilde{\Delta}} \sum_{r, s \geq 0} 2^{-r} \Lambda^{2 s+r} z^{r-s} \sum_{p=0}^{\left[\frac{r}{2}\right]}(2 m)^{r-2 p} \\
& \times \sum_{I \vdash r} \sum_{J \vdash s}\left[G_{c, \Delta^{\prime}}^{r}\right]^{\left(2^{p}, 1^{r-2 p}\right) I}\left\langle\nu_{\Delta^{\prime}, I}\left|V_{+}(1)\right| \nu_{\tilde{\Delta}, J}\right\rangle\left[G_{c, \tilde{\Delta}}^{s}\right]^{J\left(1^{s}\right)}
\end{aligned}
$$

and

$$
\begin{aligned}
\Psi_{\iota}^{2}\left(z ; \Lambda, m_{1}, m_{2}\right)= & z^{\Delta^{\prime}-\Delta_{+}-\tilde{\Delta}} \sum_{r, s \geq 0}\left(\frac{1}{2} \Lambda\right)^{r+s} z^{r-s} \sum_{p=0}^{\left[\frac{r}{2}\right]}\left(2 m_{1}\right)^{r-2 p} \sum_{p^{\prime}=0}^{\left[\frac{s}{2}\right]}\left(2 m_{2}\right)^{s-2 p^{\prime}} \\
& \times \sum_{I \vdash r} \sum_{J \vdash s}\left[G_{c, \Delta^{\prime}}^{r}\right]^{\left(2^{p}, 1^{r-2 p}\right) I}\left\langle\nu_{\Delta^{\prime}, I}\left|V_{+}(1)\right| \nu_{\tilde{\Delta}, J}\right\rangle\left[G_{c, \tilde{\Delta}}^{s}\right]^{J\left(2^{p^{\prime}}, 1^{s-2 p^{\prime}}\right)} .
\end{aligned}
$$

In what follows it will be convenient to introduce the following notation

$$
\Psi_{\iota}^{\mathbf{i}}(z ; \Lambda, \cdot)=z^{\kappa_{\iota}} \Phi_{\iota}^{\mathbf{i}}(z ; \Lambda, \cdot), \quad \mathbf{i}=\mathbf{1}, \mathbf{2}, \quad \iota=\mathrm{I}, \mathrm{II},
$$

where

$$
\kappa_{\iota}=\Delta^{\prime}-\Delta_{+}-\tilde{\Delta}= \begin{cases}\Delta\left(\sigma+\frac{b}{4}\right)-\Delta_{+}-\Delta\left(\sigma-\frac{b}{4}\right)=-b \sigma-\Delta_{+} & \text {if } \quad \iota=\mathrm{I} \\ \Delta\left(\sigma-\frac{b}{4}\right)-\Delta_{+}-\Delta\left(\sigma+\frac{b}{4}\right)=b \sigma-\Delta_{+} & \text {if } \quad \iota=\text { II. }\end{cases}
$$

Let us notice that $\Phi_{\iota}^{\mathbf{i}}(z ; \Lambda, \cdot)$ can be split into "diagonal" $r=s$ and "off-diagonal" $r \neq s$ parts $\Phi_{\iota}^{\mathbf{i}}(z ; \Lambda, \cdot)=\Phi_{\iota, r=s}^{\mathbf{i}}(\Lambda, \cdot)+\Phi_{\iota, r \neq s}^{\mathbf{i}}(z ; \Lambda, \cdot)$. The former does not depend on $z$ leaving this dependence entirely to the latter. Making use of this observation $\Psi_{\iota}^{\mathrm{i}}(z ; \Lambda, \cdot)$ can be cast into the factorized form

$$
\Psi_{\iota}^{\mathrm{i}}(z ; \Lambda, \cdot)=z^{\kappa_{\iota}} \mathrm{e}^{\mathcal{Y}_{\iota}^{\mathrm{i}}(\Lambda, \cdot)} \mathrm{e}^{\mathcal{X}_{\iota}^{\mathrm{i}}(z ; \Lambda, \cdot)}, \quad \mathbf{i}=\mathbf{1}, \mathbf{2}, \quad \iota=\mathrm{I}, \mathrm{II} .
$$

The functions in the exponent take the form

$$
\mathcal{Y}_{\iota}^{\mathbf{i}}(\Lambda, \cdot)=\log \Phi_{\iota, r=s}^{\mathbf{i}}(\Lambda, \cdot), \quad \mathcal{X}_{\iota}^{\mathbf{i}}(z ; \Lambda, \cdot)=\log \left(1+\frac{\Phi_{\iota, r \neq s}^{\mathbf{i}}(z ; \Lambda, \cdot)}{\Phi_{\iota, r=s}^{\mathbf{i}}(\Lambda, \cdot)}\right) .
$$

The factorized form in eq. (3.4), as we soon see, gives one some insight into the behavior of $\Psi_{\iota}^{\mathbf{i}}$ in the classical limit. The equations (2.35D1) and (2.35D2) under substitution (3.2) take the form

$$
\left[\frac{1}{b^{2}} z^{2} \frac{\partial^{2}}{\partial z^{2}}+\left(\frac{2 \kappa_{\iota}}{b^{2}}-\frac{5-\mathbf{i}}{4-\mathbf{i}}\right) z \frac{\partial}{\partial z}+\frac{\Lambda}{4-\mathbf{i}} \frac{\partial}{\partial \Lambda}+\mathcal{U}_{\iota}^{\mathbf{i}}(z ; \Lambda, \cdot)\right] \Phi_{\iota}^{\mathbf{i}}(z ; \Lambda, \cdot)=0,
$$

where $\mathbf{i}=\mathbf{1}, \mathbf{2}, \iota=\mathrm{I}$, II and

$$
\begin{aligned}
\mathcal{U}_{\iota}^{1}(z ; \Lambda, m) & =\frac{\kappa_{\iota}\left(\kappa_{\iota}-1\right)}{b^{2}}-\frac{4 \kappa_{\iota}}{3}+\frac{1}{4} z^{2} \Lambda^{2}+z m \Lambda+\frac{\Lambda^{2}}{z}+\frac{2 \tilde{\Delta}+\Delta^{\prime}-\Delta_{+}}{3}, \\
\mathcal{U}_{\iota}^{2}\left(z ; \Lambda, m_{1}, m_{2}\right) & =\frac{\kappa_{\iota}\left(\kappa_{\iota}-1\right)}{b^{2}}-\frac{3 \kappa_{\iota}}{2}+\frac{1}{4} \Lambda^{2}\left(z^{2}+\frac{1}{z^{2}}\right)+\Lambda\left(z m_{1}+\frac{m_{2}}{z}\right)+\frac{\tilde{\Delta}+\Delta^{\prime}-\Delta_{+}}{2} .
\end{aligned}
$$


Let us consider the limit $b \rightarrow 0$ of eqs. (3.5). To this purpose it is convenient to replace the parameter $\sigma$ in $\Delta^{\prime}$ and $\tilde{\Delta}$ with $\xi=b \sigma$, cf. (2.28). Recall that the weights $\Delta^{\prime}, \tilde{\Delta}$ are related by the fusion rules written in eqs. (2.29) and (2.30). Hence, in the limit $b \rightarrow 0$

- the conformal weights in eqs. (3.5) read as follows

$$
\begin{aligned}
\Delta^{\prime}, \tilde{\Delta} & \stackrel{b \rightarrow 0}{\sim} \frac{1}{b^{2}} \delta, \quad \text { where } \quad \delta=\lim _{b \rightarrow 0} b^{2} \Delta^{\prime}=\lim _{b \rightarrow 0} b^{2} \tilde{\Delta}=\frac{1}{4}-\xi^{2}, \\
\Delta_{+} & \stackrel{b \rightarrow 0}{\sim} \mathcal{O}\left(b^{0}\right) \quad \text { and } \quad 2 \tilde{\Delta}+\Delta^{\prime}-\Delta_{+} \stackrel{b \rightarrow 0}{\sim} \frac{1}{b^{2}} 3 \delta, \quad \tilde{\Delta}+\Delta^{\prime}-\Delta_{+} \stackrel{b \rightarrow 0}{\sim} \frac{1}{b^{2}} 2 \delta ;
\end{aligned}
$$

— the $\kappa_{\iota}$ 's yield (cf. (3.3))

$$
\begin{gathered}
\kappa_{\iota} \stackrel{b \rightarrow 0}{\longrightarrow}\left\{\begin{array}{r}
-\xi+\frac{1}{2} \text { if } \iota=\mathrm{I} \\
\xi+\frac{1}{2} \text { if } \iota=\mathrm{II}
\end{array}\right. \\
\kappa_{\iota}\left(\kappa_{\iota}-1\right) \stackrel{b \rightarrow 0}{\longrightarrow}-\left(\frac{1}{4}-\xi^{2}\right)=-\delta \quad \text { for } \iota=\mathrm{I}, \mathrm{II} .
\end{gathered}
$$

Making use of the relationship between CFT and instanton parameters given in eqs. (2.16), (2.17) that reveals their $b$ dependence i.e., $\Lambda=\hat{\Lambda} /\left(\epsilon_{1} b\right), m=m_{1}=\hat{m}_{1} /\left(\epsilon_{1} b\right), m_{2}=$ $\hat{m}_{2} /\left(\epsilon_{1} b\right)$, and taking into account the fact that the external conformal weights $\Delta^{\prime}, \tilde{\Delta}$ are heavy in the classical limit, while the degenerate weight $\Delta_{+}$labeling the degenerate vertex operator is light, we conjecture that the degenerate irregular blocks given in eqs. $(2.24 \Psi 1)$ and $(2.24 \Psi 2)$ factorize into the light and the heavy parts in the classical limit. For $\Phi_{\iota}^{\mathbf{i}}=$ $z^{-\kappa_{\iota}} \Psi_{\iota}^{\mathbf{i}}$ this assertion translates into the following asymptotic behavior in the limit $b \rightarrow 0$

$$
\Phi_{\iota}^{\mathrm{i}}\left(z ; \hat{\Lambda} /\left(\epsilon_{1} b\right), \cdot\right) \stackrel{b \rightarrow 0}{\sim} \varphi_{\iota}^{\mathrm{i}}\left(z ; \hat{\Lambda} / \epsilon_{1}, \cdot\right) \mathrm{e}^{\frac{1}{b^{2}} f_{\delta}^{\mathrm{i}}\left(\hat{\Lambda} / \epsilon_{1}, \cdot\right)}, \quad \mathbf{i}=\mathbf{1}, \mathbf{2}, \quad \iota=\mathrm{I}, \mathrm{II} .
$$

Comparing the right hand sides of eqs. (3.6) to eqs. (3.4) one can expect that

$$
\begin{aligned}
\varphi_{\iota}^{\mathrm{i}}\left(z ; \hat{\Lambda} / \epsilon_{1}, \cdot\right) & =\lim _{b \rightarrow 0} \mathrm{e}^{\mathcal{X}_{\iota}^{\mathrm{i}}(z ; \Lambda, \cdot)}=\lim _{b \rightarrow 0}\left(1+\frac{\Phi_{\iota, r \neq s}^{\mathbf{i}}\left(z ; \hat{\Lambda} /\left(\epsilon_{1} b\right), \cdot\right)}{\Phi_{\iota, r=s}^{\mathbf{i}}\left(\hat{\Lambda} /\left(\epsilon_{1} b\right), \cdot\right)}\right), \\
f_{\delta}^{\mathrm{i}}\left(\hat{\Lambda} / \epsilon_{1}, \cdot\right) & =\lim _{b \rightarrow 0} b^{2} \mathcal{Y}_{\iota}^{\mathbf{i}}(\Lambda, \cdot)=\lim _{b \rightarrow 0} b^{2} \log \Phi_{\iota, r=s}^{\mathbf{i}}\left(\hat{\Lambda} /\left(\epsilon_{1} b\right), \cdot\right)
\end{aligned}
$$

In ref. [23], where the similar discussion was performed for the $N_{f}=0$ case to obtain the Mathieu equation, we were able to prove this conjecture keeping only dominating factors in $b^{-2}$ and neglecting all the sub-dominating ones. Although much complex, a similar proof, in principle, could be performed in the case under study. We expect, however, similar results, therefore, in what follows, we are content with the assumption of the factorization (3.6) as a fact.

Taking into account the factorization (3.6), in the limit $b \rightarrow 0$ from eqs. (3.5) one gets

- for $\iota=\mathrm{I}$ :

$$
\left[z^{2} \frac{\mathrm{d}^{2}}{\mathrm{~d} z^{2}}+2\left(\frac{1}{2}-\xi\right) z \frac{\mathrm{d}}{\mathrm{d} z}+\mathscr{U}^{\mathbf{i}}\left(z ; \hat{\Lambda} / \epsilon_{1}, \cdot\right)+\frac{\hat{\Lambda}}{4-\mathbf{i}} \partial_{\hat{\Lambda}} f_{\delta}^{\mathbf{i}}\left(\hat{\Lambda} / \epsilon_{1}, \cdot\right)\right] \varphi_{\mathrm{I}}^{\mathbf{i}}\left(z ; \hat{\Lambda} / \epsilon_{1}, \cdot\right)=0,
$$


- for $\iota=$ II:

$$
\left[z^{2} \frac{\mathrm{d}^{2}}{\mathrm{~d} z^{2}}+2\left(\frac{1}{2}+\xi\right) z \frac{\mathrm{d}}{\mathrm{d} z}+\mathscr{U}^{\mathbf{i}}\left(z ; \hat{\Lambda} / \epsilon_{1}, \cdot\right)+\frac{\hat{\Lambda}}{4-\mathbf{i}} \partial_{\hat{\Lambda}} f_{\delta}^{\mathbf{i}}\left(\hat{\Lambda} / \epsilon_{1}, \cdot\right)\right] \varphi_{\mathrm{II}}^{\mathbf{i}}\left(z ; \hat{\Lambda} / \epsilon_{1}, \cdot\right)=0,
$$

where $\mathbf{i}=\mathbf{1}, \mathbf{2}$ and

$$
\begin{aligned}
\mathscr{U}^{1}\left(z ; \frac{\hat{\Lambda}}{\epsilon_{1}}, m\right) & =\frac{1}{4} \frac{\hat{\Lambda}^{2}}{\epsilon_{1}^{2}} z^{2}+\frac{\hat{\Lambda} \hat{m}}{\epsilon_{1}^{2}} z+\frac{\hat{\Lambda}^{2}}{\epsilon_{1}^{2}} \frac{1}{z}, \\
\mathscr{U}^{2}\left(z ; \frac{\hat{\Lambda}}{\epsilon_{1}}, m_{1}, m_{2}\right) & =\frac{1}{4} \frac{\hat{\Lambda}^{2}}{\epsilon_{1}^{2}}\left(z^{2}+\frac{1}{z^{2}}\right)+\frac{\hat{\Lambda}}{\epsilon_{1}}\left(\frac{\hat{m}_{1}}{\epsilon_{1}} z+\frac{\hat{m}_{2}}{\epsilon_{1}} \frac{1}{z}\right) .
\end{aligned}
$$

The classical blocks $f_{\delta}^{\mathbf{1}}, f_{\delta}^{\mathbf{2}}$ are defined in eqs. (2.19) and (2.20). Deriving the above equations (3.8) and (3.9) we assumed that $b^{2} \hat{\Lambda} \partial_{\hat{\Lambda}} \varphi_{\iota}^{\mathbf{i}} \rightarrow 0$ for $b \rightarrow 0$. This conjecture was verified by direct computations of $\hat{\Lambda} / \epsilon_{1}$ expansion of $\varphi_{\iota}^{\mathbf{i}}$ (see below). Although it has not been proved, in analogy to the $N_{f}=0$ case in ref. [23], where it was proved up to leading order in $b^{-2}$ that $\varphi^{\mathbf{0}}$ is independent of $b$, it is here expected that $\varphi_{\iota}^{\mathbf{i}}$ also does not depend on $b$ as well as its $\hat{\Lambda}$ derivative.

In order to get rid of the first order differential operator in eqs. (3.8) and (3.9) we redefine the functions $\varphi_{\mathrm{I}}^{\mathbf{i}}, \varphi_{\mathrm{II}}^{\mathbf{i}}$ :

$$
\varphi_{\mathrm{I}}^{\mathbf{i}}=z^{\xi} \psi_{\mathrm{I}}^{\mathbf{i}}, \quad \varphi_{\mathrm{II}}^{\mathbf{i}}=z^{-\xi} \psi_{\mathrm{II}}^{\mathbf{i}}
$$

and subsequently change a variable $z=\mathrm{e}^{w}$. In result we obtain

$$
\left[\frac{\mathrm{d}^{2}}{\mathrm{~d} w^{2}}+\mathscr{U}^{\mathbf{i}}\left(\mathrm{e}^{w} ; \hat{\Lambda} / \epsilon_{1}, \cdot\right)+\frac{\hat{\Lambda}}{4-\mathbf{i}} \partial_{\hat{\Lambda}} f_{\delta}^{\mathbf{i}}\left(\hat{\Lambda} / \epsilon_{1}, \cdot\right)-\xi^{2}\right] \psi_{\mathrm{I}}^{\mathbf{i}}\left(\mathrm{e}^{w} ; \hat{\Lambda} / \epsilon_{1}, \cdot\right)=0, \quad \mathbf{i}=\mathbf{1}, \mathbf{2}
$$

and the same equations for the second solutions $\psi_{\mathrm{II}}^{\mathbf{i}}\left(\mathrm{e}^{w} ; \hat{\Lambda} / \epsilon_{1}, \cdot\right)$. Potentials $\mathscr{U}^{\mathbf{i}}$ are defined

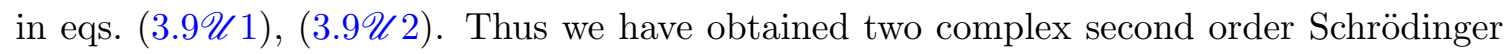
type differential equations with spectra $\lambda_{\xi}^{\mathbf{i}} / 4:=\xi^{2}-\hat{\Lambda} \partial_{\hat{\Lambda}} f_{\delta}^{\mathbf{i}} /(4-\mathbf{i}), \mathbf{i}=\mathbf{1}, \mathbf{2}$ and pairs $\left(\psi_{\mathrm{I}}^{\mathbf{i}}, \psi_{\mathrm{II}}^{\mathbf{i}}\right)$ of independent solutions. Below we address each of the case $N_{f}=1$ and $N_{f}=2$ separately, where we compute the spectra and eigenfunctions of relevant operators.

\subsection{Single flavor case: solvable complex potential}

In this subsection we narrow down the discussion to $N_{f}=1$ case. In order to obtain the differential equation for this case that takes the form of Hill's equation (1.1), but that possesses a complex periodic potential, we identify the "coupling constant" as $h=2 \hat{m} / \epsilon_{1}=$ $2 \hat{\Lambda} / \epsilon_{1}$ in eq. (3.11) with potential (3.9 U 1$)$ and, after the change of variable $w=-2 i x$, we obtain $^{16}$

$$
\left[-\frac{\mathrm{d}^{2}}{\mathrm{~d} x^{2}}+\frac{h^{2}}{4} \mathrm{e}^{-4 i x}+2 h^{2} \cos 2 x\right] \psi^{\mathbf{1}}=\lambda_{\xi}^{\mathbf{1}} \psi^{\mathbf{1}}, \quad x \in \mathbb{R} / \pi \mathbb{Z} .
$$

\footnotetext{
${ }^{16} \mathrm{~A}$ reason we choose this particular change of variable is dictated by experience gained in ref. [23], where this choice led to the coincidence of the expansion of pure gauge three-point degenerate irregular block with similar "weak coupling" expansion of the Mathieu exponent me $e_{\nu}(x)$.
} 
This is the Schrödinger equation with complex odd potential on unit circle. The eigenvalue $\lambda_{\xi}^{\mathbf{1}}$ can be obtained from eqs. (2.21a) and (2.21b) with parametric relation to Floquet's exponent $\xi=\nu / 2$,

$$
\begin{aligned}
\lambda_{\nu}^{\mathbf{1}}(h) & =\nu^{2}-\frac{4 h}{3} \partial_{h} f_{\frac{1}{4}\left(1-\nu^{2}\right)}^{\mathbf{1}}\left(\frac{1}{2} h, \frac{1}{2} h\right) \\
& =\nu^{2}+\frac{2}{3\left(\nu^{2}-1\right)} h^{4}+\frac{3}{32\left(\nu^{2}-4\right)\left(\nu^{2}-1\right)} h^{6}+\frac{5 \nu^{2}+7}{8\left(\nu^{2}-4\right)\left(\nu^{2}-1\right)^{3}} h^{8}+\mathcal{O}\left(h^{10}\right) .
\end{aligned}
$$

The expression (3.13) is well defined for $\nu \notin \mathbb{Z}$. The first few terms in the expansion (3.13) suggest that the spectrum $\lambda_{\nu}^{\mathbf{1}}(h)$ is real for $h \in \mathbb{R}$ and $\nu \in \mathbb{R} \backslash \mathbb{Z}$. Indeed, from our approach easily follows that the quantity $\lambda_{\nu}^{\mathbf{1}}(h)$ is real-valued for $b, \hat{\Lambda}, \hat{m}, \epsilon_{1}, \xi \in \mathbb{R}$. The eigenvalue $\lambda_{\nu}^{\mathbf{1}}(h)$ in eq. (3.13) is given by the logarithmic derivative of the $N_{f}=1$ classical irregular block:

$$
f_{\delta}^{1}\left(\hat{\Lambda} / \epsilon_{1}, \hat{m} / \epsilon_{1}\right)=\lim _{b \rightarrow 0} b^{2} \log \mathcal{F}_{1+6 Q^{2}, \frac{1}{b^{2}} \delta}\left(\hat{\Lambda} /\left(\epsilon_{1} b\right), \hat{m} /\left(\epsilon_{1} b\right)\right) .
$$

Let us recall that the $N_{f}=1$ quantum irregular block $\mathcal{F}_{c, \Delta}(\Lambda, m)$ is defined as an expansion in $\Lambda$ and $m$ with coefficients which are elements of the inverse of the Gram matrix, i.e., rational functions of $c$ and $\Delta$. Therefore, $\mathcal{F}_{c, \Delta}(\Lambda, m)$ is real-valued function when all its parameters:

$$
c=1+6 Q^{2}, \quad \Delta=\frac{1}{b^{2}} \delta=\frac{1}{b^{2}}\left(\frac{1}{4}-\xi^{2}\right), \quad \Lambda=\hat{\Lambda} /\left(\epsilon_{1} b\right), \quad m=\hat{m} /\left(\epsilon_{1} b\right)
$$

are real. Hence, in particular, for $b, \hat{\Lambda}, \hat{m}, \epsilon_{1}, \xi \in \mathbb{R}$ and $\mathcal{F}_{c, \Delta}(\Lambda, m) \geq 0$ the function $\log \mathcal{F}_{c, \Delta}(\Lambda, m)$ is real-valued. If $\mathcal{F}_{c, \Delta}(\Lambda, m)<0$ then the function $\log \mathcal{F}_{c, \Delta}(\Lambda, m)$ will have constant imaginary part $i \pi$ which vanishes in the limit $b \rightarrow 0$.

In our approach the corresponding eigenfunctions, i.e. two independent solutions of eq. (3.12) can be given in the "weak coupling" expansion (small $h$ ). The eigenfunctions are calculable from the classical limit of the "off-diagonal" part of $\Phi_{\iota, r \neq s}^{\mathbf{1}}$ given in eq. (3.1 1 1) (see also eqs. $(3.7 \varphi)$ and $(3.10)$ ) in the same manner as in ref. [23]. As a result one finds ${ }^{17}$

$$
\begin{aligned}
\psi_{\mathrm{I}}^{\mathbf{1}} \equiv \psi_{\mathrm{I}}^{\mathbf{1}}\left(\mathrm{e}^{-2 i x} ; \frac{1}{2} h, \frac{1}{2} h\right) & =\mathrm{e}^{i \nu x} \lim _{b \rightarrow 0}\left(1+\frac{\Phi_{\mathrm{I}, r \neq s}^{\mathbf{1}}\left(\mathrm{e}^{-2 i x} ; \frac{h}{2 b}, \frac{h}{2 b}\right)}{\Phi_{\mathrm{I}, r=s}^{\mathbf{1}}\left(\frac{h}{2 b}, \frac{h}{2 b}\right)}\right) \\
& =\mathrm{e}^{i \nu x}+\sum_{n \geq 1} \mathcal{R}_{\mathrm{I}, n}^{\mathbf{1}}(x ; \nu)\left(\frac{h}{2}\right)^{2 n},
\end{aligned}
$$

where three first coefficients are found to take the form

$$
\begin{aligned}
\mathcal{R}_{\mathrm{I}, 1}^{1}(x ; \nu)= & \frac{\mathrm{e}^{(\nu-2) i x}}{\nu-1}-\frac{\mathrm{e}^{(\nu+2) i x}}{\nu+1}+\frac{\mathrm{e}^{(\nu-4) i x}}{8(\nu-2)} \\
\mathcal{R}_{\mathrm{I}, 2}^{1}(x ; \nu)= & \frac{1}{2}\left(\frac{(5-\nu) \mathrm{e}^{(\nu-2) i x}}{4(\nu-2)\left(\nu^{2}-1\right)}+\frac{\mathrm{e}^{(\nu-4) i x}}{(\nu-2)(\nu-1)}+\frac{\mathrm{e}^{(\nu+4) i x}}{(\nu+1)(\nu+2)}-\frac{(5-3 \nu) \mathrm{e}^{(\nu-6) i x}}{12(\nu-3)(\nu-2)(\nu-1)}\right. \\
& \left.+\frac{\mathrm{e}^{(\nu-8) i x}}{64(\nu-4)(\nu-2)}\right)
\end{aligned}
$$

${ }^{17}$ Here, $\xi=\nu / 2$. 


$$
\begin{aligned}
\mathcal{R}_{\mathrm{I}, 3}^{1}(x ; \nu)= & \frac{1}{2}\left(\frac{\left(\nu^{2}-4 \nu+7\right) \mathrm{e}^{(\nu-2) i x}}{(\nu-2)(\nu-1)^{3}(\nu+1)}-\frac{\left(\nu^{2}+4 \nu+7\right) \mathrm{e}^{(\nu+2) i x}}{(\nu-1)(\nu+1)^{3}(\nu+2)}+\frac{2 \mathrm{e}^{(\nu-4) i x}}{3(\nu-3)(\nu-2)\left(\nu^{2}-1\right)}\right. \\
& -\frac{\mathrm{e}^{(\nu+6) i x}}{3(\nu+1)(\nu+2)(\nu+3)}+\frac{\mathrm{e}^{(\nu-6) i x}}{3(\nu-3)(\nu-2)(\nu-1)} \\
& +\frac{(9-\nu) \mathrm{e}^{(\nu-6) i x}}{64(\nu-4)(\nu-2)(\nu-1)(\nu+1)}+\frac{(3 \nu-7) \mathrm{e}^{(\nu-8) i x}}{6(\nu-4)(\nu-3)(\nu-2)(\nu-1)} \\
& \left.+\frac{\left(15 \nu^{2}-80 \nu+89\right) \mathrm{e}^{(\nu-10) i x}}{960(\nu-5)(\nu-4)(\nu-3)(\nu-2)(\nu-1)}+\frac{\mathrm{e}^{(\nu-12) i x}}{1536(\nu-6)(\nu-4)(\nu-2)}\right),
\end{aligned}
$$

and so on. Analogously, the second solution that solves eq. (3.12) is given by the formula:

$$
\psi_{\mathrm{II}}^{\mathbf{1}} \equiv \psi_{\mathrm{II}}^{\mathbf{1}}\left(\mathrm{e}^{-2 i x} ; \frac{1}{2} h, \frac{1}{2} h\right)=\mathrm{e}^{-i \nu x} \lim _{b \rightarrow 0}\left(1+\frac{\Phi_{\mathrm{II}, r \neq s}^{\mathbf{1}}\left(\mathrm{e}^{-2 i x} ; \frac{h}{2 b}, \frac{h}{2 b}\right)}{\Phi_{\mathrm{II}, r=s}^{\mathbf{1}}\left(\frac{h}{2 b}, \frac{h}{2 b}\right)}\right) .
$$

We have found no examples in the literature for this equation and its solutions which we could compare with.

\subsection{Two flavors case: Whittaker-Hill equation}

In $N_{f}=2$ case, after change of variable $w=-2 i x$ in eq. (3.11) for $\mathbf{i}=2$ with poten-

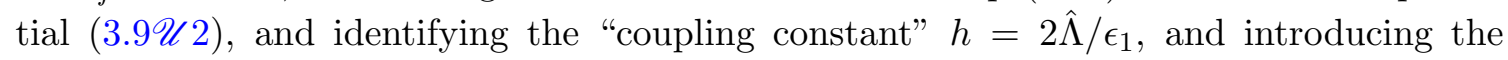
second parameter $\mu=\hat{m}_{1} / \epsilon_{1}=\hat{m}_{2} / \epsilon_{1}$ the equation assumes the form:

$$
\left[-\frac{\mathrm{d}^{2}}{\mathrm{~d} x^{2}}+\frac{1}{2} h^{2} \cos 4 x+4 h \mu \cos 2 x\right] \psi^{2}=\lambda_{\xi}^{2} \psi^{2},
$$

which is a specific example of the Hill equation (1.1) termed the Whittaker-Hill equation. The eigenvalue $\lambda_{\xi}^{2}$, after the identification $\xi=\nu / 2$, is given by the following formula (cf. (2.22a) and (2.22b))

$$
\begin{aligned}
\lambda_{\nu}^{2}(h, \mu)= & \nu^{2}-2 h \partial_{h} f_{\frac{1}{4}\left(1-\nu^{2}\right)}^{2}\left(\frac{1}{2} h, \mu, \mu\right) \\
= & \nu^{2}+\frac{2}{\nu^{2}-1} \mu^{2} h^{2}+\left(\frac{1}{2\left(\nu^{2}-4\right)}+\frac{12}{\left(\nu^{2}-1\right)\left(\nu^{2}-4\right)} \mu^{2}+\frac{8\left(5 \nu^{2}+7\right)}{\left(\nu^{2}-4\right)\left(\nu^{2}-1\right)^{3}} \mu^{4}\right) \frac{h^{4}}{16} \\
& +\left(\frac{20}{\left(\nu^{2}-9\right)\left(\nu^{2}-4\right)\left(\nu^{2}-1\right)} \mu^{2}+\frac{32\left(7 \nu^{2}+17\right)}{\left(\nu^{2}-9\right)\left(\nu^{2}-4\right)\left(\nu^{2}-1\right)^{3}} \mu^{4}\right. \\
& \left.+\frac{64\left(9 \nu^{4}+58 \nu^{2}+29\right)}{\left(\nu^{2}-9\right)\left(\nu^{2}-4\right)\left(\nu^{2}-1\right)^{5}} \mu^{6}\right) \frac{h^{6}}{64}+\mathcal{O}\left((h / 2)^{8}\right) .
\end{aligned}
$$

Hence, as before the $2 \mathrm{dCFT}$ technics led to the so-called non-integer order $(\nu \notin \mathbb{Z})$ solution of the Whittaker-Hill equation. The above expression for $\lambda_{\nu}^{2}$ exactly coincides with that obtained in ref. [24] by means of the perturbation calculus (see [19]) applied to eq. (3.16). 
As in the previous case, the two independent solutions of eq. (3.16) can be given in the "weak coupling" expansions and are computed from the classical limit of the "off-diagonal"

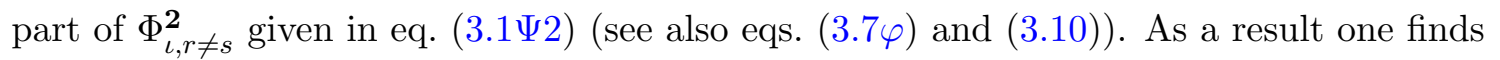

$$
\begin{aligned}
\psi_{\mathrm{I}}^{2} & \equiv \psi_{\mathrm{I}}^{2}\left(\mathrm{e}^{-2 i x} ; \frac{1}{2} h, \mu, \mu\right) \\
& =\mathrm{e}^{i \nu x} \lim _{b \rightarrow 0}\left(1+\frac{\Phi_{\mathrm{I}, r \neq s}^{2}\left(\mathrm{e}^{-2 i x} ; \frac{h}{2 b}, \frac{\mu}{b}, \frac{\mu}{b}\right)}{\Phi_{\mathrm{I}, r=s}^{2}\left(\frac{h}{2 b}, \frac{\mu}{b}, \frac{\mu}{b}\right)}\right)=\mathrm{e}^{i \nu x}+\sum_{n \geq 1} \mathcal{R}_{\mathrm{I}, n}^{2}(x ; \nu, \mu)\left(\frac{h}{2}\right)^{2 n},
\end{aligned}
$$

where the first tree coefficients of the Whittaker-Hill function take the form:

$$
\begin{aligned}
& \mathcal{R}_{\mathrm{I}, 1}^{2}(x ; \nu, \mu)=\mu\left(\frac{\mathrm{e}^{-i x(\nu+2)}}{\nu-1}-\frac{\mathrm{e}^{-i x(\nu-2)}}{\nu+1}\right), \\
& \mathcal{R}_{\mathrm{I}, 2}^{2}(x ; \nu, \mu)=\frac{\mathrm{e}^{-i x(\nu+4)}}{8(\nu-2)}-\frac{\mathrm{e}^{-i x(\nu-4)}}{8(\nu+2)}+\frac{1}{2} \mu^{2}\left(\frac{\mathrm{e}^{-i x(\nu-4)}}{(\nu+1)(\nu+2)}+\frac{\mathrm{e}^{-i x(\nu+4)}}{(\nu-2)(\nu-1)}\right), \\
& \mathcal{R}_{\mathrm{I}, 3}^{2}(x ; \nu, \mu)=\mu\left(-\frac{(\nu-5) \mathrm{e}^{-i x(\nu+2)}}{8(\nu-2)(\nu-1)(\nu+1)}-\frac{(\nu+5) \mathrm{e}^{-i x(\nu-2)}}{8(\nu-1)(\nu+1)(\nu+2)}\right. \\
& \left.+\frac{(3 \nu-5) \mathrm{e}^{-i x(\nu+6)}}{24(\nu-3)(\nu-2)(\nu-1)}+\frac{(3 \nu+5) \mathrm{e}^{-i x(\nu-6)}}{24(\nu+1)(\nu+2)(\nu+3)}\right) \\
& +\mu^{3}\left(\frac{\left(\nu^{2}-4 \nu+7\right) \mathrm{e}^{-i x(\nu+2)}}{2(\nu-2)(\nu-1)^{3}(\nu+1)}-\frac{\left(\nu^{2}+4 \nu+7\right) \mathrm{e}^{-i x(\nu-2)}}{2(\nu-1)(\nu+1)^{3}(\nu+2)}\right. \\
& \left.+\frac{\mathrm{e}^{-i x(\nu+6)}}{6(\nu-3)(\nu-2)(\nu-1)}-\frac{\mathrm{e}^{-i x(\nu-6)}}{6(\nu+1)(\nu+2)(\nu+3)}\right) .
\end{aligned}
$$

In order to obtain the second solution it suffices to take

$$
\psi_{\mathrm{II}}^{2} \equiv \psi_{\mathrm{II}}^{2}\left(\mathrm{e}^{-2 i x} ; \frac{1}{2} h, \mu, \mu\right)=\mathrm{e}^{-i \nu x} \lim _{b \rightarrow 0}\left(1+\frac{\Phi_{\mathrm{II}, r \neq s}^{2}\left(\mathrm{e}^{-2 i x} ; \frac{h}{2 b}, \frac{\mu}{b}, \frac{\mu}{b}\right)}{\Phi_{\mathrm{II}, r=s}^{2}\left(\frac{h}{2 b}, \frac{\mu}{b}, \frac{\mu}{b}\right)}\right) .
$$

In analogy to the Mathieu's exponent one can term the above solutions generalized Mathieu exponents or Whittaker-Hill exponents.

\section{Conclusions}

In the present paper we have shown that the $N_{f}=1$ and $N_{f}=2$ classical irregular blocks solve the Schrödinger eigenvalue problem for the complex (1.4) and Whittaker-Hill (1.3) potentials, respectively. ${ }^{18}$ In addition, working entirely within the CFT framework we have derived for each of the above cases the corresponding two linearly independent solutions. These eigenfunctions are determined by two different fusion rules imposed on the in- and

\footnotetext{
${ }^{18}$ Such observation in the case of the Whittaker-Hill operator has been already made in [24]. The $N_{f}=1$ case has not been discussed in the literature so far.
} 
out-states conformal weights appearing in the degenerate irregular blocks, in accordance with the Feigin-Fuchs Theorem.

The conformal field theory setup proved to be especially powerful in the study of the eigenvalue problem for the complex potential (1.4). For $x \in \mathbb{R}$ the potential (1.4) is $\pi$-periodic and the corresponding quantum-mechanical hamiltonian is evidently PTsymmetric. It turned out that such hamiltonian in the weak coupling region (small $h \in \mathbb{R}$ ) and for the real non-integer values of the Floquet parameter $\nu \notin \mathbb{Z}$ has a real spectrum determined by the $N_{f}=1$ classical irregular block expansion. From the definition of the $N_{f}=1$ classical irregular block it immediately follows that the spectrum is real for $h \in \mathbb{R}$ and $\nu \in \mathbb{R} \backslash \mathbb{Z} .^{19}$ Therefore, we have found yet another new example of the PT-invariant hamiltonian with complex periodic potential which has a real spectrum. Such hamiltonians have interesting applications in the branch of the condensed matter theory known as "complex crystals" (see e.g. [13, 49]). They also provide models for testing postulates of the PT-symmetric quantum mechanics (cf. [13] and refs. therein).

As has been already mentioned our solutions to eqs. (3.12) and (3.16) make sense for small $h \in \mathbb{R}$ and $\nu \notin \mathbb{Z}$. Hence, two interesting questions arise at this point: (i) How within $2 \mathrm{dCFT}$ one can get the solutions in the other regions of the spectrum, in particular, for large coupling constant(s)? (ii) How is it possible to derive from the irregular blocks the solutions with integer values of the Floquet parameter? Work is in progress in order to answer these questions.

As a final remark let us stress that it is possible to extend results of the present paper to generic situation when $N_{f}=$ odd/even number of flavors, cf. [24, 25]. The case with an even number of flavors should lead to the solution of the equation termed in [24] as "generalized Mathieu equation", i.e., Schrödinger equation with potential built out of higher cosine terms. In the same way the NVD equations obeyed by the three-point degenerate irregular blocks with an odd number of flavors will produce in the classical limit solutions of the Schrödinger equation with potential being generalization of (1.4). The latter case seems to be an interesting task for further investigation due its possible application as a laboratory for study of implications of PT symmetry.

\section{A Floquet's theorem and band structure of spectrum}

In this appendix we recall the most important facts concerning the eq. (1.1). Let $y_{i}(x)=$ $y_{i}(x ; \lambda), i=1,2$ be the normalized fundamental solutions of $(1.1)$, i.e., such that $y_{1}(0)=$

\footnotetext{
${ }^{19}$ Let us note that an alternate reality proof of the spectrum of PT-symmetric hamiltonian $\mathcal{H}_{1}$ should be possible to carry out at the gauge theory side of the AGT correspondence. The $\mathcal{N}=2$, SYM counterpart of the $N_{f}=1$ classical irregular block is known as the $N_{f}=1, \mathrm{SU}(2)$ effective twisted superpotential. This quantity is determined by a solution of certain Bethe-like equation appearing as a saddle point condition in the process of computation of the Nekrasov-Shatashvili limit of the corresponding Nekrasov instanton partition function, cf. [22, 47, 48]. Thus, in order to prove the reality (and the positivity) of the spectrum in eq. (3.12) it seems to be enough to find an answer to the following question: in what circumstances is the logarithmic derivative of the $N_{f}=1, \mathrm{SU}(2)$ effective twisted superpotential real valued (and positive), or perhaps equivalently, in what circumstances the corresponding saddle point/Bethe-like equation has real (and positive) solution? Finally, let us stress that very similar "Bethe-ansatz-inspired" ideas were employed to prove the reality and the positivity properties of the spectra of PT-symmetric Schrödinger operators emerging in the context of ODE/IM correspondence, cf. [29].
} 
$y_{2}^{\prime}(0)=1$ and $y_{2}(0)=y_{1}^{\prime}(0)=0$. The periodicity of $Q(x)$ implies that $y(x+\pi)$ is also a solution of eq. (1.1). The fundamental solutions $y_{i}(x), i=1,2$ satisfy the following equation

$$
v(x+\pi)=v(x) M(\lambda), \quad v=\left(\begin{array}{ll}
y_{1} & y_{2}
\end{array}\right), \quad M(\lambda):=\left(\begin{array}{cc}
y_{1}(\pi ; \lambda) & y_{2}(\pi ; \lambda) \\
y_{1}^{\prime}(\pi ; \lambda) & y_{2}^{\prime}(\pi ; \lambda)
\end{array}\right),
$$

where $M(\lambda)$ is the monodromy matrix. Wronskian for the normalized fundamental solutions amounts to $W\left(y_{1}, y_{2}\right)=1$ which entails that $M(\lambda) \in \operatorname{SL}(2, \mathbb{C})$. Since $y(x)$ is a linear combination of fundamental solutions and $y(x+\pi)$ also belongs to the set of all solutions to eq. (1.1) it is possible to pick such one, that $y(x+\pi)=\rho y(x)$. This amounts to the diagonalisation of monodromy matrix

$$
\begin{aligned}
\chi(\rho) & :=\operatorname{det}(M(\lambda)-\rho I)=\rho^{2}-\Delta(\lambda) \rho+1, \\
\chi\left(\rho_{1}\right) & =\chi\left(\rho_{2}\right)=0, \quad \rho_{1}:=\mathrm{e}^{i \nu \pi}, \quad \rho_{2}:=\mathrm{e}^{-i \nu \pi}, \quad \nu \in \mathbb{C},
\end{aligned}
$$

where $\Delta(\lambda):=\operatorname{tr} M(\lambda)$ is the Hill's discriminant, $\rho_{1}, \rho_{2}$ are the Floquet multipliers and $\nu$ is the Floquet's exponent.

Theorem 2 (Floquet). Let $\rho_{1}, \rho_{2}$ be the roots of $\chi(\rho)$.

1. If $\rho_{1} \neq \rho_{2}$ then

$$
f_{1}(x)=e^{i \nu x} p_{1}(x), \quad f_{2}(x)=e^{-i \nu x} p_{2}(x), \quad p_{i}(x+\pi)=p_{i}(x), \quad i=1,2
$$

are the two solutions that span the space of solutions to Hill's equation (1.1).

2. If $\rho_{1}=\rho_{2}$ the monodromy matrix $M(\lambda)$ is similar to upper-triangular matrix, that is there exists a solution $p(x)$ which is either periodic or anti-periodic $(p(x+\pi)=-p(x))$ and the second linearly independent solution $q(x)$ such, that

$$
q(x+\pi)=\rho_{1} q(x)+\alpha p(x) .
$$

In case $\alpha=0$ the monodromy matrix $M(\lambda) \in \mathbb{Z}_{2}$, i.e., $y_{2}(\pi)=y_{1}^{\prime}(\pi)=0$ and all solutions are either periodic or anti-periodic.

Note, that if $\nu \in \mathbb{C}$ with $\Im \nu \neq 0$ the solutions are unbounded to which one refers as to unstable solutions. In order to have stable solutions it is necessary for the Floquet exponent $\nu \in \mathbb{R}$. However, it is insufficient condition as follows from the first part of the second point of the Floquet theorem. A sufficient condition is $\nu \in \mathbb{R}$ and $M(\lambda) \in \mathbb{Z}_{2}$ which entails that $\nu=1,2$. In this case all solutions are stable and periodic with the basic period $\pi$ or $2 \pi$. An effective criterion for the stability of solutions to Hill's equation is $\Delta(\lambda) \in \mathbb{R}$ and either $|\Delta(\lambda)|<2$ or $|\Delta(\lambda)|=2$ and $M(\lambda) \in \mathbb{Z}_{2}$. From eq. (A.1) it follows that $\Delta(\lambda)=y_{1}(\pi ; \lambda)+y_{2}^{\prime}(\pi ; \lambda)$. Therefore, solving $\Delta(\lambda)= \pm 2$ for $\lambda$ we obtain segments on $\mathbb{R}_{+}$ where $|\Delta(\lambda)|<2$ as well as those, where $|\Delta(\lambda)|>2$. The former are termed bands whereas the latter - gaps. Thus, the spectrum of Hill's equation (1.1) has the band structure. This fact is a thesis of the following: 
Theorem 3 (Oscillation). Let $\lambda \in \mathbb{C}$ and $\underline{\lambda}_{i}, \bar{\lambda}_{i} \in \mathbb{R}_{+}$such that $\Delta\left(\underline{\lambda}_{i}\right)=2$ for $i \in \mathbb{N}_{0}$ and $\Delta\left(\bar{\lambda}_{i}\right)=-2$ for $i \in \mathbb{N}$. Then solutions to eq. (1.1) are stable if

$$
\lambda \in \bigcup_{i \in \mathbb{N}}\left(\underline{\lambda}_{2 i-2}, \bar{\lambda}_{2 i-1}\right) \cup\left(\bar{\lambda}_{2 i}, \underline{\lambda}_{2 i-1}\right),
$$

and in case when $\lambda=\bar{\lambda}_{2 i-1}=\bar{\lambda}_{2 i}$ or $\lambda=\underline{\lambda}_{2 i-1}=\underline{\lambda}_{2 i}$ for $i \in I \subset \mathbb{N}$ and unstable otherwise.

Open Access. This article is distributed under the terms of the Creative Commons Attribution License (CC-BY 4.0), which permits any use, distribution and reproduction in any medium, provided the original author(s) and source are credited.

\section{References}

[1] W. Magnus and S. Winkler, Hill's equation, Interscience tracts in pure and applied mathematics, Interscience, New York U.S.A. (1966).

[2] E. Mathieu, Mémoire sur le mouvement vibratoire d'une membrane de forme elliptique, J. Math. Pures Appl. 13 (1868) 137.

[3] N.W. McLachlan, Theory and application of Mathieu functions, Clarendon Press, Oxford (1947).

[4] W. Paul, Electromagnetic traps for charged and neutral particles, Rev. Mod. Phys. 62 (1990) 531 [INSPIRE].

[5] L.F. Roncaratti and V. Aquilanti, Whittaker-Hill equation, Ince polynomials, and molecular torsional modes, Int. J. Quantum Chem. 110 (2010) 716.

[6] Y. Cho, N. Kan and K. Shiraishi, Compactification in deconstructed gauge theory with topologically nontrivial link fields, Acta Phys. Polon. B 35 (2004) 1597 [hep-th/0306012] [INSPIRE].

[7] J. Lachapelle, A study of the growth of entropy fluctuations during reheating in Roulette inflation, MSc Thesis, McGill University (2008).

[8] J. Lachapelle and R.H. Brandenberger, Preheating with Non-Standard Kinetic Term, JCAP 04 (2009) 020 [arXiv: 0808.0936] [INSPIRE].

[9] S.S. Gubser and A. Hashimoto, Exact absorption probabilities for the D3-brane, Commun. Math. Phys. 203 (1999) 325 [hep-th/9805140] [InSPIRE].

[10] R. Manvelyan, H.J.W. Muller-Kirsten, J.Q. Liang and Y.-b. Zhang, Absorption cross-section of scalar field in supergravity background, Nucl. Phys. B 579 (2000) 177 [hep-th/0001179] [INSPIRE].

[11] D.K. Park, S.N. Tamarian, H.J.W. Muller-Kirsten and J.-z. Zhang, D-branes and their absorptivity in Born-Infeld theory, Nucl. Phys. B 594 (2001) 243 [hep-th/0005165] [INSPIRE].

[12] B.B. Srivastava, Fundamentals of Nuclear Physics, Rastogi Publications, Meerut India (2011).

[13] C.M. Bender, Making sense of non-Hermitian Hamiltonians, Rept. Prog. Phys. 70 (2007) 947 [hep-th/0703096] [INSPIRE]. 
[14] N. Wyllard, $A_{N-1}$ conformal Toda field theory correlation functions from conformal $\mathcal{N}=2$ $\mathrm{SU}(N)$ quiver gauge theories, JHEP 11 (2009) 002 [arXiv:0907.2189] [INSPIRE].

[15] R. Poghossian, Deformed $S W$ curve and the null vector decoupling equation in Toda field theory, JHEP 04 (2016) 070 [arXiv:1601.05096] [InSPIRE].

[16] H. Poghosyan, R. Poghossian and G. Sarkissian, The light asymptotic limit of conformal blocks in Toda field theory, JHEP 05 (2016) 087 [arXiv:1602.04829] [INSPIRE].

[17] G. Poghosyan and R. Poghossian, VEV of Baxter's Q-operator in $N=2$ gauge theory and the BPZ differential equation, arXiv: 1602.02772 [INSPIRE].

[18] E. Whittaker and G. Watson, A course of modern analysis, Cambridge Mathematical Library, Cambridge University Press (1996).

[19] H. Müller-Kirsten, Introduction to Quantum Mechanics: Schrödinger Equation and Path Integral, World Scientific (2006).

[20] A.-K. Kashani-Poor and J. Troost, Pure $\mathcal{N}=2$ super Yang-Mills and exact WKB, JHEP 08 (2015) 160 [arXiv: 1504.08324] [INSPIRE].

[21] K. Maruyoshi and M. Taki, Deformed Prepotential, Quantum Integrable System and Liouville Field Theory, Nucl. Phys. B 841 (2010) 388 [arXiv:1006.4505] [InSPIRE].

[22] M. Piatek and A.R. Pietrykowski, Classical irregular block, $\mathcal{N}=2$ pure gauge theory and Mathieu equation, JHEP 12 (2014) 032 [arXiv:1407.0305] [INSPIRE].

[23] M. Piatek and A.R. Pietrykowski, Classical limit of irregular blocks and Mathieu functions, JHEP 01 (2016) 115 [arXiv: 1509.08164] [INSPIRE].

[24] C. Rim and H. Zhang, Classical Virasoro irregular conformal block, JHEP 07 (2015) 163 [arXiv: 1504.07910] [INSPIRE].

[25] G. Bonelli, K. Maruyoshi and A. Tanzini, Wild Quiver Gauge Theories, JHEP 02 (2012) 031 [arXiv: 1112.1691] [INSPIRE].

[26] G. Başar and G.V. Dunne, Resurgence and the Nekrasov-Shatashvili limit: connecting weak and strong coupling in the Mathieu and Lamé systems, JHEP 02 (2015) 160 [arXiv: 1501.05671] [INSPIRE].

[27] P. Dorey and R. Tateo, Anharmonic oscillators, the thermodynamic Bethe ansatz and nonlinear integral equations, J. Phys. A 32 (1999) L419 [hep-th/9812211] [INSPIRE].

[28] V.V. Bazhanov, S.L. Lukyanov and A.B. Zamolodchikov, Spectral determinants for Schrödinger equation and $Q$ operators of conformal field theory,

J. Statist. Phys. 102 (2001) 567 [hep-th/9812247] [inSPIRE].

[29] P. Dorey, C. Dunning and R. Tateo, The ODE/IM Correspondence,

J. Phys. A 40 (2007) R205 [hep-th/0703066] [InSPIRE].

[30] C. Rim and H. Zhang, Classical Virasoro irregular conformal block II, JHEP 09 (2015) 097 [arXiv: 1506.03561] [INSPIRE].

[31] V.G. Kac, Contravariant Form for Infinite Dimensional Lie Algebras and Superalgebras, in Group Theoretical Methods In Physics, W. Beiglbock, A. Bohm and E. Takasugi eds., Springer-Verlag (1978), pp. 441-445.

[32] B.L. Feigin and D.B. Fuks, Invariant skew symmetric differential operators on the line and Verma modules over the Virasoro algebra, Funct. Anal. Appl. 16 (1982) 114 [InSPIRE]. 
[33] B. Feigin and D. Fuchs, Representations of the Virasoro algebra, in Representations of Lie groups and related topics, Advanced Studies in Contemporary Mathematics, A.M. Vershik and D.P. Zhelobenko eds., volume 7, Gordon and Breach, London U.K. (1990), pp. 465-554.

[34] C.B. Thorn, Computing the Kac Determinant Using Dual Model Techniques and More About the No-Ghost Theorem, Nucl. Phys. B 248 (1984) 551 [INSPIRE].

[35] V.G. Kac and A.K. Raina, Bombay Lectures on Highest Weight Representations of Infinite Dimensionsal Lie Algebras, volume 2, World Scientific (1987).

[36] D. Gaiotto, Asymptotically free $\mathcal{N}=2$ theories and irregular conformal blocks, J. Phys. Conf. Ser. 462 (2013) 012014 [arXiv:0908.0307] [InSPIRE].

[37] A. Marshakov, A. Mironov and A. Morozov, On non-conformal limit of the AGT relations, Phys. Lett. B 682 (2009) 125 [arXiv:0909.2052] [INSPIRE].

[38] E. Felinska, Z. Jaskolski and M. Kosztolowicz, Whittaker pairs for the Virasoro algebra and the Gaiotto-Bonelli-Maruyoshi-Tanzini states, J. Math. Phys. 53 (2012) 033504 [Erratum ibid. 53 (2012) 129902] [arXiv:1112.4453] [INSPIRE].

[39] D. Gaiotto and J. Teschner, Irregular singularities in Liouville theory and Argyres-Douglas type gauge theories, JHEP 12 (2012) 050 [arXiv:1203.1052] [INSPIRE].

[40] V. Alba and A. Morozov, Non-conformal limit of AGT relation from the 1-point torus conformal block, JETP Lett. 90 (2009) 708 [arXiv:0911.0363] [INSPIRE].

[41] L. Hadasz, Z. Jaskolski and P. Suchanek, Proving the AGT relation for $N_{f}=0,1,2$ antifundamentals, JHEP 06 (2010) 046 [arXiv: 1004.1841] [INSPIRE].

[42] M.-C. Tan, M-Theoretic Derivations of 4d-2d Dualities: From a Geometric Langlands Duality for Surfaces, to the AGT Correspondence, to Integrable Systems, JHEP 07 (2013) 171 [arXiv: 1301.1977] [INSPIRE].

[43] D. Maulik and A. Okounkov, Quantum Groups and Quantum Cohomology, arXiv: 1211.1287 [INSPIRE].

[44] N.A. Nekrasov and S.L. Shatashvili, Quantization of Integrable Systems and Four Dimensional Gauge Theories, arXiv:0908.4052 [INSPIRE].

[45] J. Teschner, Liouville theory revisited, Class. Quant. Grav. 18 (2001) R153 [hep-th/0104158] [INSPIRE].

[46] A.A. Belavin, A.M. Polyakov and A.B. Zamolodchikov, Infinite Conformal Symmetry in Two-Dimensional Quantum Field Theory, Nucl. Phys. B 241 (1984) 333 [inSPIRE].

[47] M.R. Piatek and A.R. Pietrykowski, Irregular blocks, $\mathcal{N}=2$ gauge theory and Mathieu system, J. Phys. Conf. Ser. 670 (2016) 012041 [INSPIRE].

[48] F. Ferrari, M.R. Piatek and A.R. Pietrykowski, $2 d$ CFT/Gauge/Bethe correspondence and solvable quantum-mechanical systems, J. Phys. Conf. Ser. 670 (2016) 012022 [InSPIRE].

[49] C.M. Bender, G.V. Dunne and P.N. Meisinger, Complex periodic potentials with real band spectra, Phys. Lett. A 252 (1999) 272 [cond-mat/9810369] [INSPIRE]. 